\title{
Numerical Algorithms for the Fractional Diffusion-Wave Equation with Reaction Term
}

\author{
Hengfei Ding and Changpin Li \\ Department of Mathematics, Shanghai University, Shanghai 200444, China \\ Correspondence should be addressed to Changpin Li; lcp@shu.edu.cn
}

Received 24 April 2013; Revised 24 June 2013; Accepted 24 June 2013

Academic Editor: Juan J. Trujillo

Copyright (C) 2013 H. Ding and C. Li. This is an open access article distributed under the Creative Commons Attribution License, which permits unrestricted use, distribution, and reproduction in any medium, provided the original work is properly cited.

\begin{abstract}
Two numerical algorithms are derived to compute the fractional diffusion-wave equation with a reaction term. Firstly, using the relations between Caputo and Riemann-Liouville derivatives, we get two equivalent forms of the original equation, where we approximate Riemann-Liouville derivative by a second-order difference scheme. Secondly, for second-order derivative in space dimension, we construct a fourth-order difference scheme in terms of the hyperbolic-trigonometric spline function. The stability analysis of the derived numerical methods is given by means of the fractional Fourier method. Finally, an illustrative example is presented to show that the numerical results are in good agreement with the theoretical analysis.
\end{abstract}

\section{Introduction}

The realm of fractional differential equations has drawn increasing attention and interest due to their important applications in biology, physics, chemistry, biochemistry, medicine, and engineering [1-8]. Generally speaking, the analytical solutions of most fractional differential equations are not easy, even impossible, to obtain, so seeking numerical solutions of these equations becomes more and more important. Till now, there have been some studies in this respect. For example, Cui [9] proposed a compact finite difference method for the fractional diffusion equation. Chen et al. [10] analyzed an implicit difference scheme for the subdiffusion equation and proved the unconditional stability and convergence. Li et al. [11] constructed some novel methods for the fractional calculus and fractional ordinary differential equation. Liu et al. [12] considered the numerical method and analytical technique for the modified anomalous subdiffusion equation with a nonlinear source term. Sousa [13] gave three finite difference schemes for a fractional advection diffusion problem. In [14], she furthermore presented an improved difference scheme to enhance the spatial accuracy. H. Wang and K. Wang [15] developed a fast finite difference method for the fractional diffusion equations. Yuste and Acedo [16] derived an explicit finite difference scheme and gave a new von
Neumann-type stability analysis for the fractional diffusion equations. Roop and his coworkers pioneered in considering the fractional differential equation by using the finite element method in [17-19], where the temporal derivative is the classical derivative and the spatial derivative is the fractional derivative. $\mathrm{Li}$ et al. [20] used the finite difference/finite element mixed method to numerically solve the nonlinear subdiffusion and superdiffusion partial differential equation, where both temporal and spatial derivatives are fractional derivatives. Zhang et al. [21] obtained a numerical method for the symmetric space fractional partial differential equations by using Galerkin finite element method and a backward difference technique. In [22], Zheng et al. established a fully discrete approximation for the nonlinear spatial fractional Fokker-Planck equation, where the discontinuous Galerkin finite element approach was utilized in time domain and the Galerkin finite element approach was utilized in spatial domain. Yang et al. used the matrix transform method for the Riesz space fractional diffusion and advection-dispersion equations and the time and space fractional Fokker-Planck equations [23,24], respectively. Recently, the spectral method was used to approximate the fractional calculus and the fractional differential equations $[25,26]$. Very recently, Ding and $\mathrm{Li}$ [27] proposed two kinds of finite difference schemes for the reaction-subdiffusion equation. 
As far as we know, there are some numerical methods for the subdiffusion equations $[9,10,12,16]$; however, there seem to be very limited ones for the fractional diffusionwave (seldom called "superdiffusion") equation. The possible reason is that there may be no better method to analyze the stability and convergence. And in the studies available, the accuracy of the temporal direction for the subdiffusion and fractional diffusion-wave equations is often less than second order. This motivates us to construct some higher order methods.

In this paper, we study the fractional diffusion-wave equation with a reaction term in the following form:

$$
\begin{array}{r}
{ }_{C} D_{0, t}^{\alpha} u(x, t)=K_{\alpha} \frac{\partial^{2} u(x, t)}{\partial x^{2}}-C_{\alpha} u(x, t)+f(x, t), \\
0 \leq t \leq T, \quad 0<x<L .
\end{array}
$$

Roughly speaking, the previous fractional differential equation is obtained from the classical diffusion-wave equation by replacing the second-order time derivative by a fractional derivative of order $\alpha(1<\alpha<2)$. It has been proposed to deal with disordered media to comb structures, dielectrics and semiconductors, and viscoelastic problems, for example, in the description of the propagation of stress waves in viscoelastic solids; see $[28,29]$ and references therein. In particular, in order to carry out the numerical comparisons, we study the following initial boundary values:

$$
\begin{gathered}
u(x, 0)=0, \quad \frac{\partial u(x, 0)}{\partial t}=0, \quad 0<x<L, \\
u(0, t)=\varphi_{1}(t), \quad 0 \leq t \leq T, \\
u(L, t)=\varphi_{2}(t), \quad 0 \leq t \leq T
\end{gathered}
$$

where $f(x, t), \varphi_{1}(x)$, and $\varphi_{2}(x)$ are sufficiently smooth functions and $K_{\alpha}>0$ and $C_{\alpha}>0$ are diffusion and reaction coefficients, respectively. ${ }_{C} D_{0, t}^{\alpha} u(x, t)$ is the Caputo derivative of order $\alpha(1<\alpha<2)$, which will be introduced in the following.

The remainder of this paper is organized as follows. In Section 2, we present preliminary knowledge, which is necessary for our study. In Section 3, we develop two numerical methods. Meanwhile, we investigate the solvability and stability by the matrix method and fractional Fourier method, respectively. In Section 4, a numerical experiment is carried out which supports the theoretical analysis. Finally, the paper concludes with a summary in the last section.

\section{Preliminary Knowledge}

In the present section, we introduce some important definitions and lemmas which are used later on. The following definitions and lemmas can be found in [30].

Definition 1. The left Riemann-Liouville integral operator $D_{0, t}^{-\alpha}$ of order $\alpha>0$ is defined in the following form:

$$
D_{0, t}^{-\alpha} u(x, t)=\frac{1}{\Gamma(\alpha)} \int_{0}^{t} \frac{u(x, y) d y}{(t-y)^{1-\alpha}},
$$

where $\Gamma(\cdot)$ is the Euler gamma function.
Definition 2. The left Caputo derivative operator ${ }_{C} D_{0, t}^{\alpha}$ of order $\alpha>0$ is defined in the following form:

$$
{ }_{C} D_{0, t}^{\alpha} u(x, t)=\left\{\begin{array}{l}
\frac{1}{\Gamma(m-\alpha)} \int_{0}^{t} \frac{\partial^{m} u(x, y)}{\partial y^{m}} \frac{d y}{(t-y)^{\alpha-m+1}}, \\
\frac{m-1<\alpha<m \in \mathbb{Z}^{+},}{\partial t^{m} u(x, t)} \\
\alpha=m .
\end{array}\right.
$$

Definition 3. The left Riemann-Liouville derivative operator ${ }_{\mathrm{RL}} D_{0, t}^{\alpha}$ of order $\alpha>0$ is defined by

$$
\mathrm{RL}_{0, t}^{\alpha} u(x, t)=\left\{\begin{array}{l}
\frac{1}{\Gamma(m-\alpha)}\left(\frac{\partial}{\partial t}\right)^{m} \int_{0}^{t} \frac{u(x, y) d y}{(t-y)^{\alpha-m+1}} \\
\frac{m-1<\alpha<m \in \mathbb{Z}^{+}}{\frac{\partial^{m-1} u(x, t)}{\partial t^{m-1}}} \\
\alpha=m-1 .
\end{array}\right.
$$

Lemma 4. Suppose $m-1<\alpha<m \in \mathbb{Z}^{+}$, for any $t$, if $u(x, t) \in$ $C^{m-1}[0, T]$ and $\partial u^{m-1}(x, t) / \partial t^{m-1}$ is integrable on $[0, T]$ with respect to $t$, and then

$$
\begin{aligned}
{ }_{C} D_{0, t}^{\alpha} u(x, t)= & { }_{R L} D_{0, t}^{\alpha} u(x, t) \\
& -\sum_{j=0}^{m-1} \frac{\partial^{j} u(x, 0)}{\partial t^{j}} \frac{t^{j-\alpha}}{\Gamma(j+1-\alpha)} .
\end{aligned}
$$

Lemma 5 (see [31]). The eigenvalues of the tridiagonal matrix $X$ with order $n-1$

$$
X=\left(\begin{array}{cccccc}
b & a & & & & \\
c & b & a & & & \\
& \ddots & \ddots & \ddots & & \\
& & & c & b & a \\
& & & & c & b
\end{array}\right)
$$

are given by

$$
\zeta_{\iota}=b+2 a \sqrt{\frac{c}{a}} \cos \left(\frac{\pi \iota}{n}\right), \quad \iota=1,2, \ldots, n-1 .
$$

Let $x_{l}=l h(l=0,1, \ldots, M)$ and $t_{k}=k \tau(k=0,1, \ldots, N)$, where $h=L / M$ and $\tau=T / N$ are the uniform spatial and temporal mesh sizes, respectively, and $M, N$ are two positive integers.

We consider a uniform mesh $\Omega$ with nodal points $x_{l}$ on $[0, L]$, for each segment $\left[x_{l}, x_{l+1}\right](l=0,1, \ldots, M-1)$ and point $t_{k}(k=0,1, \ldots, N)$ we define the following hyperbolictrigonometric spline function $\delta_{l}\left(x, t_{k}\right)$ which has different form with the mixed spline function in [27]:

$$
\begin{array}{r}
\mathcal{S}_{l}\left(x, t_{k}\right)=a_{l} \sinh \left(\vartheta\left(x-x_{l}\right)\right)+b_{l} \cosh \left(\vartheta\left(x-x_{l}\right)\right) \\
+c_{l} \sin \left(\vartheta\left(x-x_{l}\right)\right)+d_{l}, \quad x \in\left[x_{l}, x_{l+1}\right], \\
l=0,1, \ldots, M-1, \quad k=0,1, \ldots, N
\end{array}
$$


where $a_{l}, b_{l}, c_{l}$, and $d_{l}$ are constants and $\vartheta$ is an arbitrary parameter which can improve the accuracy of the numerical method. denote

To obtain the expressions of the coefficients in (11), we

$$
\begin{array}{ll}
\mathcal{S}_{l}\left(x_{l}, t_{k}\right)=u\left(x_{l}, t_{k}\right), & \mathcal{S}_{l}\left(x_{l+1}, t_{k}\right)=u\left(x_{l+1}, t_{k}\right), \\
\mathcal{S}_{l}^{\prime \prime}\left(x_{l}, t_{k}\right)=\mathrm{Q}\left(x_{l}, t_{k}\right), & \mathcal{S}_{l}^{\prime \prime}\left(x_{l+1}, t_{k}\right)=Q\left(x_{l+1}, t_{k}\right) .
\end{array}
$$

Through the straightforward calculations, we obtain the following expressions:

$$
\begin{aligned}
a_{l}= & \frac{(1-2 \cosh (\vartheta h)) Q\left(x_{l}, t_{k}\right)+Q\left(x_{l+1}, t_{k}\right)}{2 \vartheta^{2} \sinh (\vartheta h)} \\
& +\frac{u\left(x_{l+1}, t_{k}\right)-u\left(x_{l}, t_{k}\right)}{2 \sinh (\vartheta h)}, \\
b_{l}= & \frac{Q\left(x_{l}, t_{k}\right)}{\vartheta^{2}}, \\
\mathcal{G}_{l}= & \frac{Q\left(x_{l}, t_{k}\right)-Q\left(x_{l+1}, t_{k}\right)}{2 \vartheta^{2} \sin (\vartheta h)} \\
& +\frac{u\left(x_{l+1}, t_{k}\right)-u\left(x_{l}, t_{k}\right)}{2 \sin (\vartheta h)}, \\
d_{l}= & u\left(x_{l}, t_{k}\right)-\frac{Q\left(x_{l}, t_{k}\right)}{\vartheta^{2}},
\end{aligned}
$$

in which $l=0,1, \ldots, M-1, k=0,1, \ldots, N$.

From (11), one has

$$
\begin{array}{r}
\mathcal{S}_{l-1}\left(x, t_{k}\right) \\
=a_{l-1} \sinh \left(\mathcal{Y}\left(x-x_{l-1}\right)\right)+b_{l-1} \cosh \left(\vartheta\left(x-x_{l-1}\right)\right) \\
+c_{l-1} \sin \left(\vartheta\left(x-x_{l-1}\right)\right)+d_{l-1}, \quad x \in\left[x_{l-1}, x_{l}\right], \\
\quad l=1, \ldots, M, k=0,1, \ldots, N .
\end{array}
$$

In view of the continuity of the first-order derivative at the common nodes $\left(x_{l}, u\left(x_{l}, t_{k}\right)\right)$, that is, $\delta_{l-1}^{\prime}\left(x_{l}, t_{k}\right)=\delta_{l}^{\prime}\left(x_{l}, t_{k}\right)$, we get the following relation:

$$
\begin{array}{r}
\lambda_{1} Q\left(x_{l-1}, t_{k}\right)+\lambda_{2} Q\left(x_{l}, t_{k}\right)+\lambda_{3} Q\left(x_{l+1}, t_{k}\right) \\
=\frac{1}{h^{2}}\left[\mu_{1} u\left(x_{i-1}, t_{k}\right)+\mu_{2} u\left(x_{l}, t_{k}\right)+\mu_{3} u\left(x_{l+1}, t_{k}\right)\right], \\
\quad l=1, \ldots, M-1, k=0,1, \ldots, N,
\end{array}
$$

where

$$
\begin{aligned}
& \lambda_{1}=\frac{1}{\theta^{2}}\left(\frac{\cosh (\theta)(2 \cosh (\theta)-1)}{\sinh (\theta)}-\frac{\cos (\theta)}{\sin (\theta)}-2 \sinh (\theta)\right), \\
& \lambda_{2}=\frac{1}{\theta^{2}}\left(\frac{1-3 \cosh (\theta)}{\sinh (\theta)}+\frac{1+\cos (\theta)}{\sin (\theta)}\right), \\
& \lambda_{3}=\frac{1}{\theta^{2}}\left(\frac{1}{\sinh (\theta)}-\frac{1}{\sin (\theta)}\right), \\
& \mu_{1}=-\left(\frac{\cosh (\theta)}{\sinh (\theta)}+\frac{\cos (\theta)}{\sin (\theta)}\right), \\
& \mu_{2}=\frac{1+\cosh (\theta)}{\sinh (\theta)}+\frac{1+\cos (\theta)}{\sin (\theta)}, \\
& \mu_{3}=-\left(\frac{1}{\sinh (\theta)}+\frac{1}{\sin (\theta)}\right),
\end{aligned}
$$

and $\theta=\vartheta h$.

Obviously, from the expressions of $\mu_{1}, \mu_{2}$, and $\mu_{3}$, we find that $\mu_{1}+\mu_{2}+\mu_{3}=0$.

When $\vartheta \rightarrow 0$, then $\theta \rightarrow 0$; the spline function given by (15) reduces to the ordinary cubic spline function:

$$
\begin{array}{r}
h^{2}\left[Q\left(x_{l-1}, t_{k}\right)+4 Q\left(x_{l}, t_{k}\right)+Q\left(x_{l+1}, t_{k}\right)\right] \\
=6\left[u\left(x_{l-1}, t_{k}\right)-2 u\left(x_{l}, t_{k}\right)+u\left(x_{l+1}, t_{k}\right)\right], \\
l=1, \ldots, M-1, k=0,1, \ldots, N .
\end{array}
$$

If we use $\partial^{2} u\left(x_{l-1}, t_{k}\right) / \partial x^{2}, \partial^{2} u\left(x_{l}, t_{k}\right) / \partial x^{2}$; and $\partial^{2} u\left(x_{l+1}\right.$, $\left.t_{k}\right) / \partial x^{2}$ to take the place of $Q\left(x_{l-1}, t_{k}\right), Q\left(x_{l}, t_{k}\right)$; and $\mathrm{Q}\left(x_{l+1}, t_{k}\right)$ in (15), respectively, then one gets

$$
\begin{aligned}
\lambda_{1} \frac{\partial^{2} u\left(x_{l-1}, t_{k}\right)}{\partial x^{2}}+\lambda_{2} \frac{\partial^{2} u\left(x_{l}, t_{k}\right)}{\partial x^{2}}+\lambda_{3} \frac{\partial^{2} u\left(x_{l+1}, t_{k}\right)}{\partial x^{2}} \\
=\frac{1}{h^{2}}\left[\mu_{1} u\left(x_{l-1}, t_{k}\right)+\mu_{2} u\left(x_{l}, t_{k}\right)+\mu_{3} u\left(x_{l+1}, t_{k}\right)\right] \\
\quad+\mathcal{O}\left(h^{p}\right),
\end{aligned}
$$

where $p>0$.

Using Taylor's series for (18) at $\left(x_{l}, t_{k}\right)$, we get the truncation error as follows:

$$
\begin{aligned}
E_{i}^{k}= & \left\{\frac{1}{h}\left(\mu_{1}-\mu_{3}\right) \frac{\partial}{\partial x}\right. \\
& +\frac{1}{2}\left[2\left(\lambda_{1}+\lambda_{2}+\lambda_{3}\right)-\left(\mu_{1}+\mu_{3}\right)\right] \frac{\partial^{2}}{\partial x^{2}} \\
& +\frac{h}{6}\left[6\left(\lambda_{3}-\lambda_{1}\right)-\left(\mu_{3}-\mu_{1}\right)\right] \frac{\partial^{3}}{\partial x^{3}}
\end{aligned}
$$




$$
\begin{aligned}
&+ \frac{h^{2}}{24}\left[12\left(\lambda_{1}+\lambda_{3}\right)-\left(\mu_{1}+\mu_{3}\right)\right] \frac{\partial^{4}}{\partial x^{4}} \\
&+ \frac{h^{3}}{120}\left[20\left(\lambda_{3}-\lambda_{1}\right)-\left(\mu_{3}-\mu_{1}\right)\right] \frac{\partial^{5}}{\partial x^{5}} \\
&+\left.\frac{h^{4}}{720}\left[30\left(\lambda_{1}+\lambda_{3}\right)-\left(\mu_{1}+\mu_{3}\right)\right] \frac{\partial^{6}}{\partial x^{6}}\right\} \\
& \times u\left(x_{l}, t_{k}\right)+\mathcal{O}\left(h^{p}\right) .
\end{aligned}
$$

From the previous expression, we find that we can obtain different difference schemes by choosing different parameters $\lambda_{1}, \lambda_{2}, \lambda_{3}, \mu_{1}$, and $\mu_{2}$. In this paper, we take $\lambda_{1}=\lambda_{3}, \lambda_{2}=$ $10 \lambda_{1}$, and $\mu_{1}=\mu_{3}=12 \lambda_{1}$, and then $p=4$. Under this assumption, (18) can be rewritten as

$$
\frac{\partial^{2} u\left(x_{l}, t_{k}\right)}{\partial x^{2}}=\frac{1}{h^{2}} \frac{12 \delta_{x}^{2} u\left(x_{l}, t_{k}\right)}{12+\delta_{x}^{2}}+\mathcal{O}\left(h^{4}\right),
$$

which is the same as in [32], where $\delta_{x}^{2}$ is the second-order central difference operator with respect to $x$.

\section{Numerical Algorithms}

In the present section, we introduce two schemes for the fractional diffusion-wave equation (1) together with the homogeneous initial value conditions (2) and boundary values conditions (3) and (4).

3.1. Numerical Algorithm I. Based on Lemma 4, (1) can be written as

$$
\begin{aligned}
\mathrm{RL}_{0, t}^{\alpha} u(x, t)= & K_{\alpha} \frac{\partial^{2} u(x, t)}{\partial x^{2}}-C_{\alpha} u(x, t)+f(x, t) \\
& +\sum_{j=0}^{1} \frac{\partial^{j} u(x, 0)}{\partial t^{j}} \frac{t^{j-\alpha}}{\Gamma(j+1-\alpha)} .
\end{aligned}
$$

Applying the initial value conditions (2) yields

$$
{ }_{\mathrm{RL}} D_{0, t}^{\alpha} u(x, t)=K_{\alpha} \frac{\partial^{2} u(x, t)}{\partial x^{2}}-C_{\alpha} u(x, t)+f(x, t) .
$$

The Riemann-Liouville derivative (with homogeneous initial value conditions) can be approximated by the following scheme:

$$
{ }_{\mathrm{RL}} D_{0, t}^{\alpha} u(x, t)=\frac{1}{\tau^{\alpha}} \sum_{j=0}^{[t / \tau]} \omega_{q, j}^{(\alpha)} u(x, t-j \tau)+\mathcal{O}\left(\tau^{q}\right) .
$$

There exist various choices of the generating functions which can lead to different approximation order $q$.

Let $\omega_{q}(z, \alpha)$ be the generating function with coefficients $\omega_{q, j}^{(\alpha)}$; that is,

$$
\omega_{q}(z, \alpha)=\sum_{j=0}^{\infty} \omega_{q, j}^{(\alpha)} z^{j}
$$

If we take the generating function in the form

$$
\omega_{1}(z, \alpha)=(1-z)^{\alpha}
$$

that is,

$$
(1-z)^{\alpha}=\sum_{j=0}^{\infty} \omega_{1, j}^{(\alpha)} z^{j}
$$

then we can obtain the following first-order scheme:

$$
{ }_{\mathrm{RL}} D_{0, t}^{\alpha} u(x, t)=\frac{1}{\tau^{\alpha}} \sum_{j=0}^{[t / \tau]} \Phi_{1, j}^{(\alpha)} u(x, t-j \tau)+\mathcal{O}(\tau),
$$

in which

$$
\begin{gathered}
\omega_{1,0}^{(\alpha)}=1, \\
\omega_{1, j}^{(\alpha)}=(-1)^{j}\left(\begin{array}{c}
\alpha \\
j
\end{array}\right) \\
=(-1)^{j} \frac{\Gamma(1+\alpha)}{\Gamma(j+1) \Gamma(1+\alpha-j)}, \quad j \geq 1,
\end{gathered}
$$

and these coefficients have the following recursive relations:

$$
\omega_{1,0}^{(\alpha)}=1, \quad \omega_{1, j}^{(\alpha)}=\left(1-\frac{1+\alpha}{j}\right) \omega_{1, j-1}^{(\alpha)}, \quad j \geq 1 .
$$

If we take the generating function as

$$
\omega_{2}(z, \alpha)=\left(\frac{3}{2}-2 z+\frac{1}{2} z^{2}\right)^{\alpha},
$$

that is,

$$
\left(\frac{3}{2}-2 z+\frac{1}{2} z^{2}\right)^{\alpha}=\sum_{j=0}^{\infty} \omega_{2, j}^{(\alpha)} z^{j}
$$

which leads to order $q=2[30]$, then

$$
\mathrm{RL}_{0, t} D_{0}^{\alpha} u(x, t)=\frac{1}{\tau^{\alpha}} \sum_{j=0}^{[t / \tau]} \omega_{2, j}^{(\alpha)} u(x, t-j \tau)+\mathcal{O}\left(\tau^{2}\right) .
$$

Here we introduce two methods to determine the corresponding coefficients $\omega_{2, j}^{(\alpha)}$ of the formula (32). One of the most general methods is to use the fast Fourier transform to calculate them [30].

In the following, we give another simple but interesting method to obtain the coefficients $\omega_{2, j}^{(\alpha)}$.

By using

$$
\left(\frac{3}{2}-2 z+\frac{1}{2} z^{2}\right)^{\alpha}=\left(\frac{3}{2}\right)^{\alpha}(1-z)^{\alpha}\left(1-\frac{1}{3} z\right)^{\alpha}
$$


and (26), one gets

$$
\begin{aligned}
\left(\frac{3}{2}-2\right. & \left.z+\frac{1}{2} z^{2}\right)^{\alpha} \\
= & \left(\frac{3}{2}\right)^{\alpha}(1-z)^{\alpha}\left(1-\frac{1}{3} z\right)^{\alpha} \\
= & \left(\frac{3}{2}\right)^{\alpha}\left[\sum_{j=0}^{\infty}(-1)^{j}\left(\begin{array}{c}
\alpha \\
j
\end{array}\right) z^{j}\right]\left[\sum_{j=0}^{\infty}\left(-\frac{1}{3}\right)^{j}\left(\begin{array}{c}
\alpha \\
j
\end{array}\right) z^{j}\right] \\
= & \left(\frac{3}{2}\right)^{\alpha} \sum_{m=0}^{\infty} \sum_{n=0}^{\infty}(-1)^{m}\left(-\frac{1}{3}\right)^{n}\left(\begin{array}{c}
\alpha \\
m
\end{array}\right)\left(\begin{array}{c}
\alpha \\
n
\end{array}\right) z^{m+n} \\
= & \left(\frac{3}{2}\right)^{\alpha} \sum_{j=0}^{\infty}\left[\sum_{m=0}^{j}(-1)^{m}\left(-\frac{1}{3}\right)^{j-m}\left(\begin{array}{c}
\alpha \\
m
\end{array}\right)\left(\begin{array}{c}
\alpha \\
j-m
\end{array}\right)\right] z^{j} \\
= & \sum_{j=0}^{\infty}\left[\left(\frac{3}{2}\right)^{\alpha} \sum_{m=0}^{j}(-1)^{j}\left(\frac{1}{3}\right)^{m}\left(\begin{array}{c}
\alpha \\
m
\end{array}\right)\left(\begin{array}{c}
\alpha \\
j-m
\end{array}\right)\right] z^{j} \\
= & \sum_{j=0}^{\infty}\left[\left(\frac{3}{2}\right)^{\alpha} \sum_{m=0}^{j}\left(\frac{1}{3}\right)^{m} \omega_{1, m}^{(\alpha)} \Phi_{1, j-m}^{(\alpha)}\right] z^{j} .
\end{aligned}
$$

Comparing the previous equation with (31), one obtains

$$
\begin{aligned}
\omega_{2, j}^{(\alpha)}= & \left(\frac{3}{2}\right)^{\alpha} \sum_{m=0}^{j}\left(\frac{1}{3}\right)^{m} \omega_{1, m}^{(\alpha)} \Phi_{1, j-m}^{(\alpha)}, \\
= & (-1)^{j}\left(\frac{3}{2}\right)^{\alpha} \\
& \times \sum_{m=0}^{j}\left(\frac{1}{3}\right)^{m} \\
& \quad \times \frac{\Gamma^{2}(1+\alpha)}{m !(j-m) ! \Gamma(1+\alpha-m) \Gamma(1+\alpha+m-j)},
\end{aligned}
$$

which has the following recursive relation:

$$
\begin{gathered}
\omega_{2,0}^{(\alpha)}=\left(\frac{3}{2}\right)^{\alpha} \\
\omega_{2, j+1}^{(\alpha)} \\
=\left(\frac{3}{2}\right)^{\alpha} \sum_{m=0}^{j+1}\left(\frac{1}{3}\right)^{m} \omega_{1, m}^{(\alpha)} \omega_{1, j+1-m}^{(\alpha)} \\
=\left(\frac{3}{2}\right)^{\alpha} \sum_{m=0}^{j}\left(\frac{1}{3}\right)^{m} \omega_{1, m}^{(\alpha)} \omega_{1, j+1-m}^{(\alpha)} \\
+\left(\frac{3}{2}\right)^{\alpha}\left(\frac{1}{3}\right)^{j+1} \omega_{1, j+1}^{(\alpha)}
\end{gathered}
$$

$$
\begin{aligned}
& =\left(\frac{3}{2}\right)^{\alpha} \sum_{m=0}^{j}\left(\frac{1}{3}\right)^{m}\left(1-\frac{1+\alpha}{j+1-m}\right) \omega_{1, m}^{(\alpha)}{\omega_{1, j-m}^{(\alpha)}}^{m} \\
& +\left(\frac{3}{2}\right)^{\alpha}\left(\frac{1}{3}\right)^{j+1} \omega_{1, j+1}^{(\alpha)} \\
& =\omega_{2, j}^{(\alpha)}-(1+\alpha)\left(\frac{3}{2}\right)^{\alpha}\left(\frac{1}{3}\right)^{j} \sum_{m=0}^{j} \frac{3^{m}}{m+1} \omega_{1, m}^{(\alpha)} \Phi_{1, j-m}^{(\alpha)} \\
& +\left(\frac{3}{2}\right)^{\alpha}\left(\frac{1}{3}\right)^{j+1}\left(1-\frac{1+\alpha}{j+1}\right) \omega_{1, j}^{(\alpha)}, \quad j \geq 0 .
\end{aligned}
$$

In summary, we have the following lemma.

Lemma 6. The coefficients $\omega_{2, j}^{(\alpha)}(j=0,1, \ldots)(1<\alpha<2)$ satisfy

$$
\begin{aligned}
& \text { (1) } \omega_{2,0}^{(\alpha)}=\left(\frac{3}{2}\right)^{\alpha}>0 \text {, } \\
& \varpi_{2,1}^{(\alpha)}=-\frac{4}{3} \alpha\left(\frac{3}{2}\right)^{\alpha}<0 \text {, } \\
& \omega_{2,2}^{(\alpha)}=\frac{\alpha(8 \alpha-5)}{9}\left(\frac{3}{2}\right)^{\alpha}>0 \text {, } \\
& \omega_{2,3}^{(\alpha)}=\frac{4 \alpha(1-\alpha)(8 \alpha-7)}{81}\left(\frac{3}{2}\right)^{\alpha}<0, \\
& \omega_{2,4}^{(\alpha)}=\frac{\alpha(\alpha-1)\left(64 \alpha^{2}-176 \alpha+123\right)}{486}\left(\frac{3}{2}\right)^{\alpha}>0, \\
& \omega_{2,5}^{(\alpha)}=\frac{2 \alpha(\alpha-1)(2-\alpha)\left(64 \alpha^{2}-208 \alpha+183\right)}{3645}\left(\frac{3}{2}\right)^{\alpha} \\
& >0 \text {, } \\
& \text { (2) } \quad \sum_{j=0}^{\infty} \omega_{2, j}^{(\alpha)}=0, \quad \sum_{j=0}^{\infty}(-1)^{j} \varpi_{2, j}^{(\alpha)}=4^{\alpha} \text {. }
\end{aligned}
$$

Proof. (1) In view of (36), we easily obtain the expressions of the coefficients $\omega_{2, j}^{(\alpha)}(j=0,1, \ldots)$.

(2) In (31), if we take $z \equiv 1$, which leads to $\sum_{j=0}^{\infty} \Phi_{2, j}^{(\alpha)}=0$. Similarly, choosing $z \equiv-1$ and substitution it into (31) yield $\sum_{j=0}^{\infty}(-1)^{j} \oplus_{2, j}^{(\alpha)}=4^{\alpha}$. This completes the proof.

Finally, let $u_{l}^{k}$ be the numerical approximation of $u\left(x_{l}, t_{k}\right)$; substituting the expansions (20) and (32) in (22) and removing the higher order term, one obtains Numerical Algorithm I as follows:

$$
\begin{aligned}
\left(12 \kappa_{1}-\right. & \left.\kappa_{2}-\omega_{2,0}^{(\alpha)}\right) u_{l-1}^{k} \\
& -2\left(12 \kappa_{1}+5 \kappa_{2}+5 \omega_{2,0}^{(\alpha)}\right) u_{l}^{k}
\end{aligned}
$$




$$
\begin{aligned}
& +\left(12 \kappa_{1}-\kappa_{2}-\omega_{2,0}^{(\alpha)}\right) u_{l+1}^{k} \\
= & \omega_{2,1}^{(\alpha)}\left(u_{l-1}^{k-1}+10 u_{l}^{k-1}+u_{l+1}^{k-1}\right) \\
& +\sum_{j=2}^{k} \omega_{2, j}^{(\alpha)}\left(u_{l-1}^{k-j}+10 u_{l}^{k-j}+u_{l+1}^{k-j}\right) \\
& -\tau^{\alpha}\left(f_{l-1}^{k}+10 f_{l}^{k}+f_{l+1}^{k}\right),
\end{aligned}
$$

for $l=1, \ldots, M-1, k=1, \ldots, N$. The initial and boundary value conditions can be discretized by

$$
\begin{gathered}
u_{l}^{0}=0, \quad l=0,1, \ldots, M, \\
\frac{1}{\tau} \frac{\delta_{t} u_{l}^{0}}{1+(1 / 2) \delta_{t}}=\frac{1}{\tau} \frac{u_{l}^{1}-u_{l}^{0}}{1+(1 / 2) \delta_{t}}=0, \quad l=0,1, \ldots, M, \\
u_{0}^{k}=\varphi_{1}(k \tau), \quad u_{M}^{k}=\varphi_{2}(k \tau), \quad k=0,1, \ldots, N,
\end{gathered}
$$

where $f_{l}^{k}=f\left(x_{l}, t_{k}\right), \kappa_{1}=K_{\alpha} \tau^{\alpha} / h^{2}$, and $\kappa_{2}=C_{\alpha} \tau^{\alpha}$.

Next, we study the solvability and the local truncation error.

For convenience, denote

$$
\begin{aligned}
& \mathbf{U}^{0}=(\underbrace{0, \ldots, 0}_{M-1})^{T}, \\
& \mathbf{U}^{k}=\left(u_{1}^{k}, u_{2}^{k}, \ldots, u_{M-1}^{k}\right)^{T}, \quad k=1,2, \ldots, N, \\
& \mathbf{F}^{k}=\left(f_{1}^{k}, f_{2}^{k}, \ldots, f_{M-1}^{k}\right)^{T}, \quad k=0,1, \ldots, N, \\
& \Lambda_{1}=\left(\begin{array}{cccccc}
10 & 1 & & & & \\
1 & 10 & 1 & & & \\
& \ddots & \ddots & \ddots & & \\
& & & 1 & 10 & 1 \\
& & & & 1 & 10
\end{array}\right) \\
& \Lambda_{2}=\left(\begin{array}{cccccc}
-2 & 1 & & & & \\
1 & -2 & 1 & & & \\
& \ddots & \ddots & \ddots & & \\
& & & 1 & -2 & 1 \\
& & & & 1 & -2
\end{array}\right)
\end{aligned}
$$

Then we can give the following matrix form of the difference scheme (38):

$$
\begin{aligned}
& \left(12 \kappa_{1} \Lambda_{2}-\kappa_{2} \Lambda_{1}-\widehat{A}_{0}\right) \mathbf{U}^{k} \\
& =\widehat{A}_{1} \mathbf{U}^{k-1}+\sum_{j=2}^{k} \widehat{A}_{j} \mathbf{U}^{k-j}-\tau^{\alpha} \Lambda_{1} \mathbf{F}^{k}+\widehat{\mathbf{Y}},
\end{aligned}
$$

where

$$
\begin{gathered}
\widehat{A}_{j}=\omega_{2, j}^{(\alpha)} \Lambda_{1}, \quad j=0,1, \ldots, k, \\
\widehat{\mathbf{Y}}=(\widehat{\Psi}_{1}, \underbrace{0, \ldots, 0}_{M-3}, \widehat{\Psi}_{M-1})^{T}, \\
\widehat{\Psi}_{1}=-\left(12 \kappa_{1}-\kappa_{2}-\omega_{2,0}^{(\alpha)}\right) \varphi_{1}\left(t_{k}\right) \\
+\sum_{j=1}^{k} \omega_{2, j}^{(\alpha)} \varphi_{1}\left(t_{k-j}\right)-\tau^{\alpha} f\left(x_{0}, t_{k}\right), \\
\widehat{\Psi}_{M-1}=-\left(12 \kappa_{1}-\kappa_{2}-\omega_{2,0}^{(\alpha)}\right) \varphi_{2}\left(t_{k}\right) \\
+\sum_{j=1}^{k} \omega_{2, j}^{(\alpha)} \varphi_{2}\left(t_{k-j}\right)-\tau^{\alpha} f\left(x_{M}, t_{k}\right) .
\end{gathered}
$$

Theorem 7. The difference equation (38) is uniquely solvable.

Proof. From Lemma 5, we know that the eigenvalues of the tridiagonal matrix $\left(12 \kappa_{1} \Lambda_{2}-\kappa_{2} \Lambda_{1}-\widehat{A}_{0}\right)$ are

$$
\begin{array}{r}
\widehat{\zeta}_{l}=-48 \kappa_{1} \sin ^{2}\left(\frac{\pi l}{2 M}\right)-4\left(\kappa_{2}+\omega_{2,0}^{(\alpha)}\right)\left[3-\sin ^{2}\left(\frac{\pi l}{2 M}\right)\right], \\
l=1,2, \ldots, M-1 .
\end{array}
$$

Due to $\kappa_{1}, \kappa_{2}$, and $\omega_{2,0}^{(\alpha)}>0$, hence,

$$
\operatorname{det}\left(12 \kappa_{1} \Lambda_{2}-\kappa_{2} \Lambda_{1}-\widehat{A}_{0}\right)=\prod_{l=1}^{M-1} \widehat{\zeta}_{l} \neq 0
$$

That is, the matrix $\left(12 \kappa_{1} \Lambda_{2}-\kappa_{2} \Lambda_{1}-\widehat{A}_{0}\right)$ is nonsingular. Thus (41) is uniquely solvable, and so is (38). The proof is finished.

Theorem 8. The local truncation error of the difference scheme (38) is $\mathcal{O}\left(\tau^{2}+h^{4}\right)$.

Proof. We now define the local truncation error as

$$
\begin{aligned}
\widehat{R}_{l}^{k}= & \frac{1}{\tau^{\alpha}} \sum_{j=0}^{k} \omega_{2, j}^{(\alpha)} u\left(x_{l}, t_{k-j}\right)-\left(\frac{K_{\alpha}}{h^{2}} \frac{12 \delta_{x}^{2} u\left(x_{l}, t_{k}\right)}{12+\delta_{x}^{2}}\right) \\
& -C_{\alpha} u\left(x_{l}, t_{k}\right)-f\left(x_{l}, t_{k}\right) .
\end{aligned}
$$

Applying (20), (22), and (32), we have

$$
\begin{aligned}
\widehat{R}_{l}^{k}= & {\left[\frac{1}{\tau^{\alpha}} \sum_{j=0}^{k} \omega_{2, j}^{(\alpha)} u\left(x_{l}, t_{k-j}\right)-{ }_{\mathrm{RL}} D_{0, t}^{\alpha} u\left(x_{l}, t_{k}\right)\right] } \\
& +K_{\alpha}\left[\frac{\partial^{2} u\left(x_{l}, t_{k}\right)}{\partial x^{2}}-\left(\frac{1}{h^{2}} \frac{12 \delta_{x}^{2} u\left(x_{l}, t_{k}\right)}{12+\delta_{x}^{2}}\right)\right] \\
= & \mathcal{O}\left(\tau^{2}+h^{4}\right) .
\end{aligned}
$$

This finishes the proof. 
In the following, we discuss the stability of Numerical Algorithm I by the fractional Fourier method [16].

Let $U_{l}^{k}$ be the approximate solution of (38) and define

$$
\begin{gathered}
\varepsilon_{l}^{k}=u_{l}^{k}-U_{l}^{k}, \quad l=1,2, \ldots, M-1, k=0,1, \ldots, N, \\
\varepsilon^{k}=\left(\varepsilon_{1}^{k}, \varepsilon_{2}^{k}, \ldots, \varepsilon_{M-1}^{k}\right)^{T} .
\end{gathered}
$$

So, we can easily obtain the following round-off error equation:

$$
\begin{aligned}
\left(12 \kappa_{1}-\right. & \left.\kappa_{2}-\omega_{2,0}^{(\alpha)}\right) \varepsilon_{l-1}^{k}-2\left(12 \kappa_{1}+5 \kappa_{2}+5 \omega_{2,0}^{(\alpha)}\right) \varepsilon_{l}^{k} \\
& +\left(12 \kappa_{1}-\kappa_{2}-\omega_{2,0}^{(\alpha)}\right) \varepsilon_{l+1}^{k} \\
= & \omega_{2,1}^{(\alpha)}\left(\varepsilon_{l-1}^{k-1}+10 \varepsilon_{l}^{k-1}+\varepsilon_{l-1}^{k-1}\right) \\
& +\sum_{j=2}^{k} \omega_{2, j}^{(2-\alpha)}\left(\varepsilon_{l-1}^{k-j}+10 \varepsilon_{l}^{k-j}+\varepsilon_{l+1}^{k-j}\right) .
\end{aligned}
$$
form:

We suppose that the solution of (48) has the following

$$
\varepsilon_{l}^{k}=\xi_{k} \exp (i \phi l h),
$$

where $\phi$ is any of the real spatial wave numbers supported by the lattice. Substituting (49) into (48) gives

$$
\left[\frac{12 \kappa_{1} \sin ^{2}((1 / 2) \phi h)}{3-\sin ^{2}((1 / 2) \phi h)}+\kappa_{2}+\omega_{2,0}^{(\alpha)}\right] \xi_{k}=-\sum_{j=1}^{k} \omega_{2, j}^{(\alpha)} \xi_{k-j}
$$

If we write

$$
\xi_{k+1}=\eta \xi_{k}
$$

and assume that $\eta \equiv \eta(\phi)$ is independent of time, then we obtain the following expression of the amplification factor:

$$
\frac{12 \kappa_{1} \sin ^{2}((1 / 2) \phi h)}{3-\sin ^{2}((1 / 2) \phi h)}+\kappa_{2}=-\sum_{j=0}^{k} \omega_{2, j}^{(\alpha)} \eta^{-j} .
$$

When $|\eta|>1$ for some $\phi$, then the temporal factor of the solution grows to infinity according to (51); the numerical method is unstable. Considering the extreme value $\eta=-1$, we find that the numerical method is stable when

$$
\frac{12 \kappa_{1} \sin ^{2}((1 / 2) \phi h)}{3-\sin ^{2}((1 / 2) \phi h)}+\kappa_{2} \geq-\sum_{j=0}^{k}(-1)^{j}{\omega_{2, j}^{(\alpha)}}^{(\alpha)} \widehat{S}_{\alpha, k}
$$

Although $S_{\alpha, k}$ depends on $k$, we can estimate it by its limit value

$$
\widehat{S}_{\alpha}=\lim _{k \rightarrow \infty} \widehat{S}_{\alpha, k}=-\sum_{j=0}^{\infty}(-1)^{j} \omega_{2, j}^{(\alpha)}=-4^{\alpha} .
$$

In this case, the stability condition becomes

$$
\frac{12 \kappa_{1} \sin ^{2}((1 / 2) \phi h)}{3-\sin ^{2}((1 / 2) \phi h)}+\kappa_{2} \geq \widehat{S}_{\alpha} .
$$

Note that $\widehat{S}_{\alpha}$ is always negative, and then (55) holds for all $\kappa_{1}>0$ and $\kappa_{2}>0$. That is to say, Numerical Algorithm I (see (38)) is unconditionally stable.
3.2. Numerical Algorithm II. Differentiating (1) with order $2-\alpha$ in the sense of Riemann-Liouville yields

$$
\begin{aligned}
& { }_{\mathrm{RL}} D_{0, t}^{2-\alpha}\left({ }_{C} D_{0, t}^{\alpha} u(x, t)\right) \\
& \quad={ }_{\mathrm{RL}} D_{0, t}^{2-\alpha}\left(K_{\alpha} \frac{\partial^{2} u(x, t)}{\partial x^{2}}-C_{\alpha} u(x, t)+f(x, t)\right) .
\end{aligned}
$$

Due to

$$
{ }_{C} D_{0, t}^{\alpha} u(x, t)=D_{0, t}^{-(2-\alpha)}\left(\frac{\partial^{2} u(x, t)}{\partial t^{2}}\right)
$$

it immediately follows that

$$
\begin{aligned}
{ }_{\mathrm{RL}} D_{0, t}^{2-\alpha}\left({ }_{C} D_{0, t}^{\alpha} u(x, t)\right) & =\left({ }_{\mathrm{RL}} D_{0, t}^{2-\alpha} D_{0, t}^{-(2-\alpha)}\right)\left(\frac{\partial^{2} u(x, t)}{\partial t^{2}}\right) \\
& =\frac{\partial^{2} u(x, t)}{\partial t^{2}} .
\end{aligned}
$$

Combing (56) with (58) gives

$$
\frac{\partial^{2} u(x, t)}{\partial t^{2}}={ }_{\mathrm{RL}} D_{0, t}^{2-\alpha}\left(K_{\alpha} \frac{\partial^{2} u(x, t)}{\partial x^{2}}-C_{\alpha} u(x, t)\right)+g(x, t),
$$

where $g(x, t)={ }_{\mathrm{RL}} D_{0, t}^{2-\alpha} f(x, t)$.

Next, for the second-order time derivative $\partial^{2} u\left(x_{l}, t_{k}\right) / \partial t^{2}$, we approximate it by the usual three-point central difference formula

$$
\begin{aligned}
\frac{\partial^{2} u\left(x_{l}, t_{k}\right)}{\partial t^{2}}= & \frac{u\left(x_{l}, t_{k-1}\right)-2 u\left(x_{l}, t_{k}\right)+u\left(x_{l}, t_{k+1}\right)}{\tau^{2}} \\
& +\mathcal{O}\left(\tau^{2}\right) .
\end{aligned}
$$

Let $u_{l}^{k}$ be the numerical approximation of $u\left(x_{l}, t_{k}\right)$. Substituting the expansions (20), (32), and (60) in (59) and removing the higher-order terms, one obtains Numerical Algorithm II as follows:

$$
\begin{aligned}
u_{l-1}^{k+1}+ & 10 u_{l}^{k+1}+u_{l+1}^{k+1} \\
= & {\left[2+\omega_{2,0}^{(2-\alpha)}\left(12 \kappa_{1}-\kappa_{2}\right)\right] u_{l-1}^{k} } \\
& +2\left[10-\omega_{2,0}^{(2-\alpha)}\left(12 \kappa_{1}+5 \kappa_{2}\right)\right] u_{l}^{k}
\end{aligned}
$$




$$
\begin{aligned}
& +\left[2+\omega_{2,0}^{(2-\alpha)}\left(12 \kappa_{1}-\kappa_{2}\right)\right] u_{l+1}^{k} \\
& -u_{l-1}^{k-1}-10 u_{l}^{k-1}-u_{l+1}^{k-1} \\
& +12 \kappa_{1} \sum_{j=1}^{k} \omega_{2, j}^{(2-\alpha)}\left[u_{l-1}^{k-j}-2 u_{l}^{k-j}+u_{l+1}^{k-j}\right] \\
& -\kappa_{2} \sum_{j=1}^{k} \omega_{2, j}^{(2-\alpha)}\left[u_{l-1}^{k-j}+10 u_{l}^{k-j}+u_{l+1}^{k-j}\right] \\
& +\tau^{2}\left[g_{l-1}^{k}+10 g_{l}^{k}+g_{l+1}^{k}\right]
\end{aligned}
$$

where $l=1, \ldots, M-1, k=1, \ldots, N-1$. The initial and boundary value conditions can be discretized by

$$
\begin{gathered}
u_{l}^{0}=0, \quad l=0,1, \ldots, M, \\
\frac{1}{\tau} \frac{\delta_{t} u_{l}^{0}}{1+(1 / 2) \delta_{t}}=\frac{1}{\tau} \frac{u_{l}^{1}-u_{l}^{0}}{1+(1 / 2) \delta_{t}}=0, \quad l=0,1, \ldots, M, \\
u_{0}^{k}=\varphi_{1}(k \tau), \quad u_{M}^{k}=\varphi_{2}(k \tau), \quad k=0,1, \ldots, N,
\end{gathered}
$$

where $f_{l}^{k}=f\left(x_{l}, t_{k}\right), \kappa_{1}=K_{\alpha} \tau^{\alpha} / h^{2}$, and $\kappa_{2}=C_{\alpha} \tau^{\alpha}$.

Similar to Numerical Algorithm I, we discuss the solvability and local truncation error of Numerical Algorithm II.

Denote

$$
\mathbf{G}^{k}=\left(g_{1}^{k}, g_{2}^{k}, \ldots, g_{M-1}^{k}\right)^{T}, \quad k=0,1, \ldots, N .
$$

Then the matrix form of Numerical Algorithm II (61) is written as

$$
\begin{aligned}
\Lambda_{1} \mathbf{U}^{k+1}= & \left(2 \Lambda_{1}+\widetilde{A}_{0}\right) \mathbf{U}^{k}-\Lambda_{1} \mathbf{U}^{k-1}+\sum_{j=1}^{k} \widetilde{A}_{j} \mathbf{U}^{k-j} \\
& +\tau^{2} \Lambda_{1} \mathbf{G}^{k}+\tilde{\mathbf{Y}}, \quad k=1, \ldots, N-1,
\end{aligned}
$$

where

$$
\begin{aligned}
& \widetilde{A}_{j}=\omega_{2, j}^{(2-\alpha)}\left(12 \kappa_{1} \Lambda_{2}-\kappa_{2} \Lambda_{1}\right), \quad j=0,1, \ldots, k, \\
& \widetilde{\mathbf{Y}}=(\widetilde{\Psi}_{1}, \underbrace{0, \ldots, 0}_{M-3}, \widetilde{\Psi}_{M-1})^{T}, \\
& \widetilde{\Psi}_{1}=-\varphi_{1}\left(t_{k+1}\right)+2 \varphi_{1}\left(t_{k}\right)-\varphi_{1}\left(t_{k-1}\right) \\
& \quad+\left(12 \kappa_{1}-\kappa_{2}\right) \sum_{j=0}^{k} \omega_{2, j}^{(2-\alpha)} \varphi_{1}\left(t_{k-j}\right)+\tau^{2} g\left(x_{0}, t_{k}\right), \\
& \widetilde{\Psi}_{M-1}=-\varphi_{2}\left(t_{k+1}\right)+2 \varphi_{2}\left(t_{k}\right)-\varphi_{2}\left(t_{k-1}\right) \\
& \quad+\left(12 \kappa_{1}-\kappa_{2}\right) \sum_{j=0}^{k} \omega_{2, j}^{(2-\alpha)} \varphi_{2}\left(t_{k-j}\right)+\tau^{2} g\left(x_{M}, t_{k}\right) .
\end{aligned}
$$

Theorem 9. The difference equation (61) is uniquely solvable.
Proof. Obviously, the eigenvalues of the tridiagonal matrix $\Lambda_{1}$ are given by

$$
\widetilde{\zeta}_{l}=12-4 \sin ^{2}\left(\frac{\pi l}{2 M}\right), \quad l=1,2, \ldots, M-1 .
$$

Hence,

$$
\operatorname{det}\left(\Lambda_{1}\right)=\prod_{l=1}^{M-1} \widetilde{\zeta}_{l}>0
$$

Therefore, the solution of (61) exists and is uniquely solvable.

In order to get the local truncation error analysis, we need the following lemma.

Lemma 10. Consider

$$
\frac{1}{\tau^{2-\alpha}} \sum_{j=0}^{k}{\omega_{2, j}^{(2-\alpha)}}^{\left(2-\frac{(k \tau)^{\alpha-2}}{\Gamma(\alpha-1)}\right.}+\mathcal{O}\left(\tau^{2}\right), \quad k=1, \ldots, N .
$$

Proof. Let $u(x, t) \equiv 1$ and then from Definition 3 we have

$$
\begin{aligned}
\left.\mathrm{RL}_{0, t} D_{0-\alpha}^{2-\alpha}(x, t)\right|_{t=t_{k}} & =\left.\frac{1}{\Gamma(\alpha-1)} \frac{\partial}{\partial t} \int_{0}^{t} \frac{u(x, s)}{(t-s)^{2-\alpha}} d s\right|_{t=t_{k}} \\
& =\frac{(k \tau)^{\alpha-2}}{\Gamma(\alpha-1)} .
\end{aligned}
$$

It follows from (32) that

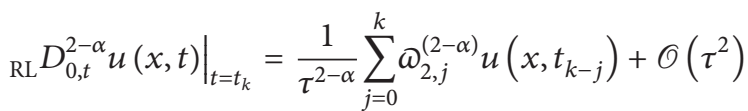

$$
\begin{aligned}
& =\frac{1}{\tau^{2-\alpha}} \sum_{j=0}^{k} \omega_{2, j}^{(2-\alpha)}+\mathcal{O}\left(\tau^{2}\right) \text {. }
\end{aligned}
$$

Combining (69) and (77) leads to

$$
\frac{1}{\tau^{2-\alpha}} \sum_{j=0}^{k} \omega_{2, j}^{(2-\alpha)}=\frac{(k \tau)^{\alpha-2}}{\Gamma(\alpha-1)}+\mathcal{O}\left(\tau^{2}\right), \quad k=1, \ldots, N .
$$

This ends the proof.

Theorem 11. The local truncation error of difference scheme (61) is $\mathcal{O}\left(\tau^{2}+h^{4}\right)$.

Proof. We now define

$$
\begin{aligned}
\widetilde{R}_{l}^{k}= & \frac{u\left(x_{l}, t_{k-1}\right)-2 u\left(x_{l}, t_{k}\right)+u\left(x_{l}, t_{k+1}\right)}{\tau^{2}} \\
& -\frac{K_{\alpha}}{\tau^{2-\alpha}} \sum_{j=0}^{k} \omega_{2, j}^{(2-\alpha)}\left(\frac{1}{h^{2}} \frac{12 \delta_{x}^{2} u\left(x_{l}, t_{k-j}\right)}{12+\delta_{x}^{2}}\right) \\
& -\frac{C_{\alpha}}{\tau^{2-\alpha}} \sum_{j=0}^{k} \omega_{2, j}^{(2-\alpha)} u\left(x_{l}, t_{k-j}\right)-g\left(x_{l}, t_{k}\right) .
\end{aligned}
$$


By using (20), (32), (59), and (60), one gets

$$
\begin{aligned}
& \widetilde{R}_{l}^{k}=\frac{u\left(x_{l}, t_{k-1}\right)-2 u\left(x_{l}, t_{k}\right)+u\left(x_{l}, t_{k+1}\right)}{\tau^{2}} \\
& -\frac{K_{\alpha}}{\tau^{2-\alpha}} \sum_{j=0}^{k} \omega_{2, j}^{(2-\alpha)}\left(\frac{1}{h^{2}} \frac{12 \delta_{x}^{2} u\left(x_{l}, t_{k-l}\right)}{12+\delta_{x}^{2}}\right) \\
& +\frac{C_{\alpha}}{\tau^{2-\alpha}} \sum_{j=0}^{k} \omega_{2, j}^{(2-\alpha)} u\left(x_{l}, t_{k-j}\right)-\frac{\partial^{2} u\left(x_{l}, t_{k}\right)}{\partial t^{2}} \\
& +K_{\alpha \mathrm{RL}} D_{0, t}^{2-\alpha}\left(\frac{\partial^{2} u\left(x_{l}, t_{k}\right)}{\partial x^{2}}\right)-C_{\alpha \mathrm{RL}} D_{0, t}^{2-\alpha} u\left(x_{l}, t_{k}\right) \\
& =\left[\frac{u\left(x_{l}, t_{k-1}\right)-2 u\left(x_{l}, t_{k}\right)+u\left(x_{l}, t_{k+1}\right)}{\tau^{2}}-\frac{\partial^{2} u\left(x_{l}, t_{k}\right)}{\partial t^{2}}\right] \\
& +\frac{K_{\alpha}}{\tau^{2-\alpha}} \sum_{j=0}^{k} \omega_{2, j}^{(2-\alpha)}\left(\frac{\partial^{2} u\left(x_{l}, t_{k-l}\right)}{\partial x^{2}}-\frac{1}{h^{2}} \frac{12 \delta_{x}^{2} u\left(x_{l}, t_{k-l}\right)}{12+\delta_{x}^{2}}\right) \\
& +K_{\alpha}\left[{ }_{\mathrm{RL}} D_{0, t}^{2-\alpha}\left(\frac{\partial^{2} u\left(x_{l}, t_{k}\right)}{\partial x^{2}}\right)\right. \\
& \left.-\frac{1}{\tau^{2-\alpha}} \sum_{j=0}^{k} \omega_{2, j}^{(2-\alpha)}\left(\frac{\partial^{2} u\left(x_{l}, t_{k-l}\right)}{\partial x^{2}}\right)\right] \\
& +C_{\alpha}\left[\frac{1}{\tau^{2-\alpha}} \sum_{j=0}^{k} \omega_{2, j}^{(2-\alpha)} u\left(x_{l}, t_{k-j}\right)-{ }_{\mathrm{RL}} D_{0, t}^{2-\alpha} u\left(x_{l}, t_{k}\right)\right] \\
& =\mathcal{O}\left(\tau^{2}\right)+\mathcal{O}\left(\tau^{2} h^{4}\right)+\frac{(k \tau)^{\alpha-2}}{\Gamma(\alpha-1)} \mathcal{O}\left(h^{4}\right) \text {. }
\end{aligned}
$$

Noticing that $1 \leq k \leq N$, then

$$
\left(\frac{1}{T}\right)^{2-\alpha} \leq(k \tau)^{\alpha-2} \leq\left(\frac{N}{T}\right)^{2-\alpha}
$$

so we can get

$$
\widetilde{R}_{l}^{k}=\sigma\left(\tau^{2}+h^{4}\right)
$$

This completes the proof.

Next, we study the stability of Numerical Algorithm II (see (61)).

As before, we can easily obtain the following round-off error equation:

$$
\begin{aligned}
\varepsilon_{l-1}^{k+1}+10 \varepsilon_{l}^{k+1}+\varepsilon_{l+1}^{k+1}= & {\left[2+\omega_{2,0}^{(2-\alpha)}\left(12 \kappa_{1}-\kappa_{2}\right)\right] \varepsilon_{l-1}^{k} } \\
& +2\left[10-\omega_{2,0}^{(2-\alpha)}\left(12 \kappa_{1}+5 \kappa_{2}\right)\right] \varepsilon_{l}^{k}
\end{aligned}
$$

$$
\begin{aligned}
& +\left[2+\omega_{2,0}^{(2-\alpha)}\left(12 \kappa_{1}-\kappa_{2}\right)\right] \varepsilon_{l+1}^{k} \\
& -\varepsilon_{l-1}^{k-1}-10 \varepsilon_{l}^{k-1}-\varepsilon_{l+1}^{k-1} \\
& +12 \kappa_{1} \sum_{j=1}^{k} \omega_{2, j}^{(2-\alpha)}\left[\varepsilon_{l-1}^{k-j}-2 \varepsilon_{l}^{k-j}+\varepsilon_{l+1}^{k-j}\right] \\
& -\kappa_{2} \sum_{j=1}^{k} \omega_{2, j}^{(2-\alpha)}\left[\varepsilon_{l-1}^{k-j}+10 \varepsilon_{l}^{k-j}+\varepsilon_{l+1}^{k-j}\right], \\
& k=1, \ldots, N-1 .
\end{aligned}
$$

Substituting (49) into (76) yields

$$
\begin{aligned}
& {\left[3-\sin ^{2}(\right.}\left.\left.\frac{1}{2} \phi h\right)\right] \xi_{k+1} \\
&=\left\{2\left[3-\sin ^{2}\left(\frac{1}{2} \phi h\right)\right]\right. \\
&-12 \kappa_{1} \omega_{2,0}^{(2-\alpha)} \sin ^{2}\left(\frac{1}{2} \phi h\right) \\
&\left.-\kappa_{2} \omega_{2,0}^{(2-\alpha)}\left[3-\sin ^{2}\left(\frac{1}{2} \phi h\right)\right]\right\} \xi_{k} \\
&- {\left[3-\sin ^{2}\left(\frac{1}{2} \phi h\right)\right] \xi_{k-1} } \\
&- \kappa_{2}\left[3-\sin ^{2}\left(\frac{1}{2} \phi h\right)\right] \\
& \times \sum_{j=1}^{k} \omega_{2, j}^{(2-\alpha)} \xi_{k-j} \\
&-12 \kappa_{1} \sin ^{2}\left(\frac{1}{2} \phi h\right) \\
& \times \sum_{j=1}^{k} \omega_{2, j}^{(2-\alpha)} \xi_{k-j} .
\end{aligned}
$$

From (51) and (77), we have

$$
\begin{aligned}
{\left[3-\sin ^{2}\left(\frac{1}{2} \phi h\right)\right] \eta=\{2} & {\left[3-\sin ^{2}\left(\frac{1}{2} \phi h\right)\right] } \\
& -12 \kappa_{1} \omega_{2,0}^{(2-\alpha)} \sin ^{2}\left(\frac{1}{2} \phi h\right) \\
& \left.-\kappa_{2} \omega_{2,0}^{(2-\alpha)}\left[3-\sin ^{2}\left(\frac{1}{2} \phi h\right)\right]\right\} \\
- & {\left[3-\sin ^{2}\left(\frac{1}{2} \phi h\right)\right] \eta^{-1} }
\end{aligned}
$$


TABLE 1: The maximum error and temporal and spatial convergence orders by Numerical Algorithm I (see (38)).

\begin{tabular}{|c|c|c|c|c|}
\hline$\alpha$ & & The maximum error & Temporal convergence order & Spatial convergence order \\
\hline \multirow{8}{*}{1.1} & $h=\frac{1}{4}, \tau=\frac{1}{2}$ & $8.057755 e-002$ & - & - \\
\hline & $h=\frac{1}{8}, \tau=\frac{1}{8}$ & $9.573435 e-003$ & 1.5366 & 3.0733 \\
\hline & $\begin{array}{ll}1 & 1\end{array}$ & & & \\
\hline & $h=\overline{16}, \tau=\overline{32}$ & $6.103333 e-004$ & 1.9857 & 3.9714 \\
\hline & $h=\frac{1}{,} \tau=1$ & $3827561 e-005$ & 19976 & 39951 \\
\hline & $n-\overline{32}, \imath-\overline{128}$ & .027 & 1.9970 & 3.9951 \\
\hline & $h=\frac{1}{r}, \tau=\frac{1}{-10}$ & $2.394875 e-006$ & 1.9992 & 3.9984 \\
\hline & $64 \quad 512$ & & & \\
\hline \multirow{7}{*}{1.3} & $h=\frac{1}{4}, \tau=\frac{1}{2}$ & $8.079213 e-002$ & - & - \\
\hline & $h=\frac{1}{8}, \tau=\frac{1}{8}$ & $1.170294 e-002$ & 1.3937 & 2.7873 \\
\hline & $\begin{array}{ll}0 & 0 \\
1 & 1\end{array}$ & & & \\
\hline & $h=\overline{16}, \tau=\overline{32}$ & $7.818506 e-004$ & 1.9519 & 3.9038 \\
\hline & $h=\frac{1}{0}, \tau=\frac{1}{10}$ & $5.015017 e-005$ & 1.9813 & 3.9626 \\
\hline & $\begin{array}{ll}32 & 128 \\
1 & 1\end{array}$ & & & \\
\hline & $h=\frac{1}{64}, \tau=\frac{1}{512}$ & $3.166977 e-006$ & 1.9925 & 3.9851 \\
\hline \multirow{8}{*}{1.5} & $h=\frac{1}{4}, \tau=\frac{1}{2}$ & $7.117932 e-002$ & - & - \\
\hline & $h=\frac{1}{0}, \tau=\frac{1}{0}$ & $1.300290 e-002$ & 1.2263 & 2.4526 \\
\hline & $\begin{array}{ll}8 & 8 \\
1 & 1\end{array}$ & & & \\
\hline & $h=\frac{1}{16}, \tau=\frac{1}{32}$ & $9.519179 e-004$ & 1.8859 & 3.7719 \\
\hline & $\begin{array}{cc}1 & 1 \\
& -1\end{array}$ & & & \\
\hline & $h=\overline{32}, \tau=\overline{128}$ & $6.385188 e-005$ & 1.9490 & 3.8980 \\
\hline & $h=\frac{1}{-}, \tau=\frac{1}{}$ & $4.135300 e-006$ & 1.9743 & 3.9487 \\
\hline & $n-\frac{64}{6},-512$ & & $1.9 / 45$ & $3.940 \%$ \\
\hline \multirow{8}{*}{1.7} & $h=\frac{1}{4}, \tau=\frac{1}{2}$ & $5.069482 e-002$ & - & 一 \\
\hline & $h=\frac{1}{-}, \tau=\frac{1}{-}$ & $1.157045 e-002$ & 1.0657 & 21314 \\
\hline & $\begin{array}{ll}8 & 8\end{array}$ & $1.1570450-002$ & & 2.1014 \\
\hline & $h=\frac{1}{\tau}, \tau=\frac{1}{00}$ & $9.766015 e-004$ & 1.7833 & 3.5665 \\
\hline & $\begin{array}{cc}16 & 32 \\
1 & 1\end{array}$ & & & \\
\hline & $h=\frac{1}{32}, \tau=\frac{1}{128}$ & $7.054045 e-005$ & 1.8956 & 3.7912 \\
\hline & $\begin{array}{cc}1 & 1 \\
h & -1\end{array}$ & & & \\
\hline & $h=\frac{1}{64}, \tau=\overline{512}$ & $4.819461 e-006$ & 1.9358 & 3.8715 \\
\hline \multirow{7}{*}{1.9} & $h=\frac{1}{4}, \tau=\frac{1}{2}$ & $1.938949 e-002$ & - & - \\
\hline & $h=\frac{1}{\mathrm{o}}, \tau=\frac{1}{\mathrm{o}}$ & $4.643245 e-003$ & 1.0310 & 2.0621 \\
\hline & $\begin{array}{ll}0 & 0 \\
1 & 1\end{array}$ & & & \\
\hline & $h=\frac{1}{16}, \tau=\frac{1}{32}$ & $5.122387 e-004$ & 1.5901 & 3.1802 \\
\hline & $h=\frac{1}{,} \tau=\frac{1}{1}$ & & & \\
\hline & $n=\overline{32}, \tau=\overline{128}$ & $4.251159 e-005$ & 1.7954 & 3.5909 \\
\hline & $h=\frac{1}{64}, \tau=\frac{1}{512}$ & $3.231450 e-006$ & 1.8588 & 3.7176 \\
\hline
\end{tabular}

$$
\begin{aligned}
& -\kappa_{2}\left[3-\sin ^{2}\left(\frac{1}{2} \phi h\right)\right] \sum_{j=1}^{k} \omega_{2, j}^{(2-\alpha)} \eta^{-j} \\
& -12 \kappa_{1} \sin ^{2}\left(\frac{1}{2} \phi h\right) \sum_{j=1}^{k} \omega_{2, j}^{(2-\alpha)} \eta^{-j}
\end{aligned}
$$

Considering the extreme value $\eta=-1$, we can obtain the following stability condition from (78):

$$
\begin{aligned}
\sin ^{2}\left(\frac{1}{2} \phi h\right) & \leq \frac{12-3 \kappa_{2}}{4+\left(12 \kappa_{1}-\kappa_{2}\right) \sum_{j=0}^{k}(-1)^{j} \omega_{2, j}^{(2-\alpha)}} \\
& \equiv \widetilde{S}_{\alpha, k} .
\end{aligned}
$$


TABLE 2: The maximum error and temporal convergence order by Numerical Scheme II (see (61)).

\begin{tabular}{|c|c|c|c|}
\hline$\alpha$ & & The maximum error & The temporal convergence order \\
\hline \multirow{4}{*}{1.1} & $h=\frac{1}{5}, \tau=\frac{1}{50}$ & $5.030598 e-004$ & ${ }_{1}$ \\
\hline & $h=\frac{1}{5}, \tau=\frac{1}{100}$ & $1.398353 e-004$ & 1.8470 \\
\hline & $h=\frac{1}{5}, \tau=\frac{1}{200}$ & $4.521910 e-005$ & 1.6287 \\
\hline & $h=\frac{1}{5}, \tau=\frac{1}{400}$ & $2.132246 e-005$ & 1.0846 \\
\hline \multirow{4}{*}{1.2} & $h=\frac{1}{6}, \tau=\frac{1}{50}$ & $4.445704 e-004$ & - \\
\hline & $h=\frac{1}{6}, \tau=\frac{1}{100}$ & $1.173968 e-004$ & 1.9210 \\
\hline & $h=\frac{1}{6}, \tau=\frac{1}{200}$ & $3.399818 e-005$ & 1.7879 \\
\hline & $h=\frac{1}{6}, \tau=\frac{1}{400}$ & $1.295545 e-005$ & 1.3919 \\
\hline \multirow{4}{*}{1.3} & $h=\frac{1}{5}, \tau=\frac{1}{50}$ & $3.816458 e-004$ & - \\
\hline & $h=\frac{1}{7}, \tau=\frac{1}{100}$ & $9.909362 e-005$ & 1.9454 \\
\hline & $h=\frac{1}{7}, \tau=\frac{1}{200}$ & $2.713495 e-005$ & 1.8686 \\
\hline & $h=\frac{1}{7}, \tau=\frac{1}{400}$ & $8.986675 e-006$ & 1.5943 \\
\hline \multirow{4}{*}{1.4} & $h=\frac{1}{5}, \tau=\frac{1}{50}$ & $3.193648 e-004$ & - \\
\hline & $h=\frac{1}{8}, \tau=\frac{1}{100}$ & $8.224534 e-005$ & 1.9572 \\
\hline & $h=\frac{1}{8}, \tau=\frac{1}{200}$ & $2.186799 e-005$ & 1.9111 \\
\hline & $h=\frac{1}{8}, \tau=\frac{1}{400}$ & $6.641262 e-006$ & 1.7193 \\
\hline \multirow{4}{*}{1.5} & $h=\frac{1}{10}, \tau=\frac{1}{50}$ & $2.574223 e-004$ & - \\
\hline & $h=\frac{1}{10}, \tau=\frac{1}{100}$ & $6.580906 e-005$ & 1.9678 \\
\hline & $h=\frac{1}{10}, \tau=\frac{1}{200}$ & $1.700153 e-005$ & 1.9526 \\
\hline & $h=\frac{1}{10}, \tau=\frac{1}{400}$ & $4.689779 e-006$ & 1.8581 \\
\hline \multirow{4}{*}{1.6} & $h=\frac{1}{15}, \tau=\frac{1}{50}$ & $1.972538 e-004$ & - \\
\hline & $h=\frac{1}{15}, \tau=\frac{1}{100}$ & $5.020982 e-005$ & 1.9740 \\
\hline & $h=\frac{1}{15}, \tau=\frac{1}{200}$ & $1.272752 e-005$ & 1.9800 \\
\hline & $h=\frac{1}{15}, \tau=\frac{1}{400}$ & $3.268637 e-006$ & 1.9612 \\
\hline \multirow{4}{*}{1.7} & $h=\frac{1}{20}, \tau=\frac{1}{50}$ & $1.402871 e-004$ & - \\
\hline & $h=\frac{1}{20}, \tau=\frac{1}{100}$ & $3.570742 e-005$ & 1.9741 \\
\hline & $h=\frac{1}{20}, \tau=\frac{1}{200}$ & $9.023412 e-006$ & 1.9845 \\
\hline & $h=\frac{1}{20}, \tau=\frac{1}{400}$ & $2.285957 e-006$ & 1.9809 \\
\hline
\end{tabular}


TABLE 2: Continued.

\begin{tabular}{rlrl}
\hline$\alpha$ & & The maximum error & The temporal convergence order \\
\hline$h$ & $=\frac{1}{20}, \tau=\frac{1}{50}$ & $8.770513 e-005$ & - \\
$h$ & $=\frac{1}{20}, \tau=\frac{1}{100}$ & $2.235014 e-005$ & 1.9724 \\
$h$ & $=\frac{1}{20}, \tau=\frac{1}{200}$ & $5.655983 e-006$ & 1.9824 \\
$h$ & $=\frac{1}{20}, \tau=\frac{1}{400}$ & $1.438349 e-006$ & 1.9754 \\
$h$ & $=\frac{1}{20}, \tau=\frac{1}{50}$ & $4.067501 e-005$ & - \\
1.9 & & $1.038309 e-005$ \\
$h$ & $=\frac{1}{20}, \tau=\frac{1}{100}$ & $2.636242 e-006$ & 1.9699 \\
$h$ & $=\frac{1}{20}, \tau=\frac{1}{200}$ & $6.778414 e-007$ & 1.9595
\end{tabular}

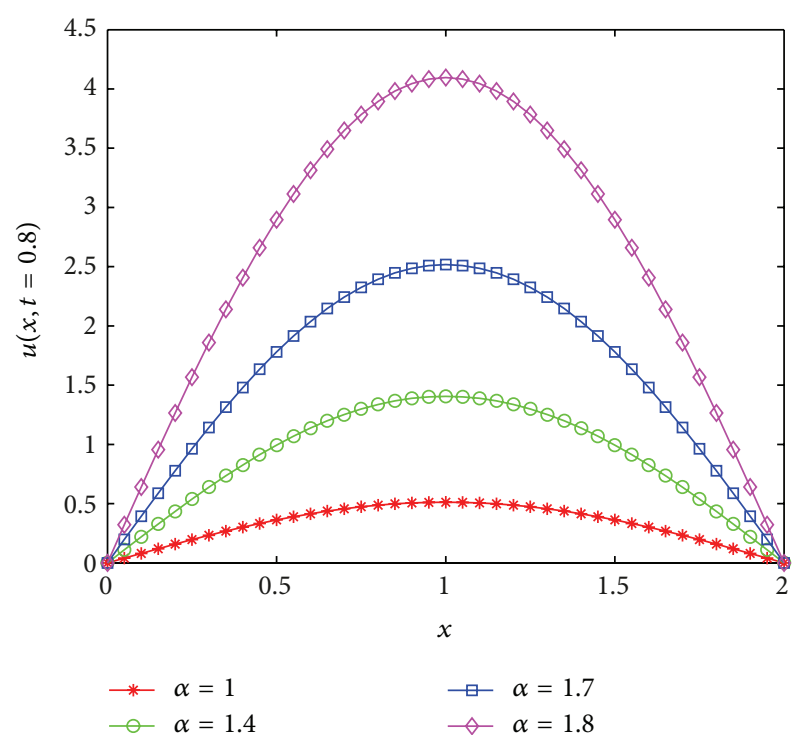

FIGURE 1: Dependence of the numerical solution for different values of $\alpha$ by Numerical Algorithm I (see (38)) with $\tau=0.002, h=$ 0.0025 .

For large enough $k$, we can estimate $\widetilde{S}_{\alpha, k}$ by its limit value

$$
\begin{aligned}
\widetilde{S}_{\alpha} & =\lim _{k \rightarrow \infty} \widetilde{S}_{\alpha, k}=\frac{12-3 \kappa_{2}}{4+\left(12 \kappa_{1}-\kappa_{2}\right) \sum_{j=0}^{\infty}(-1)^{j} \omega_{2, j}^{(2-\alpha)}} \\
& =\frac{12-3 \kappa_{2}}{4+\left(12 \kappa_{1}-\kappa_{2}\right) 4^{2-\alpha}} .
\end{aligned}
$$

It follows that

$$
\sin ^{2}\left(\frac{1}{2} \phi h\right) \leq \frac{12-3 \kappa_{2}}{4+\left(12 \kappa_{1}-\kappa_{2}\right) 4^{2-\alpha}}=\widetilde{S}_{\alpha}
$$

Because the sine function is bounded by 1 , from (81) one finds that Numerical Algorithm II is to be stable if $\widetilde{S}_{\alpha} \geq 1$,

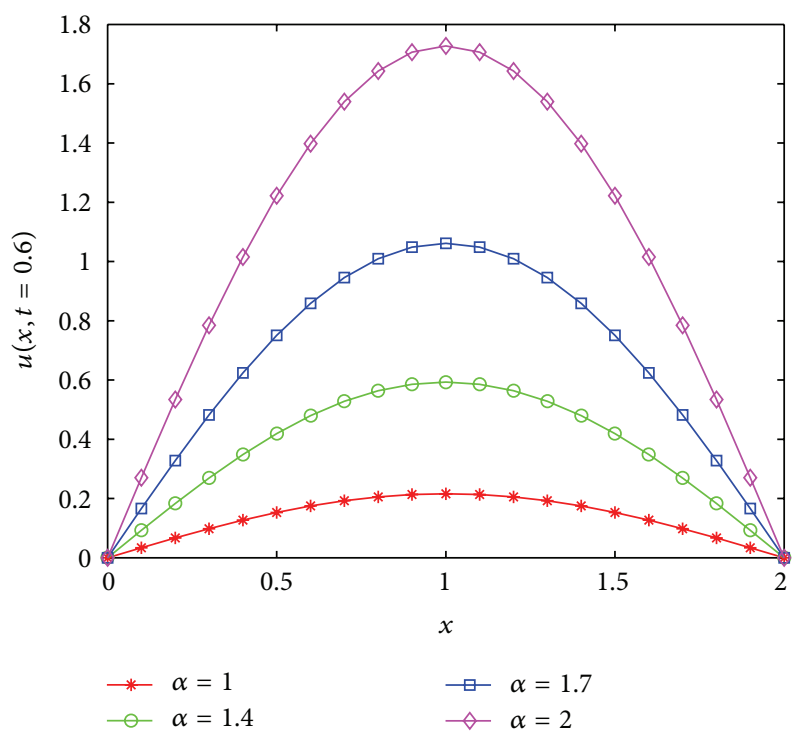

FIGURE 2: Dependence of the numerical solution for different values of $\alpha$ by Numerical Algorithm I (see (38)) with $\tau=0.0001, h=0.05$.

that is,

$$
\frac{12-3 \kappa_{2}}{4+\left(12 \kappa_{1}-\kappa_{2}\right) 4^{2-\alpha}} \geq 1
$$

Furthermore, stability condition (82) can be rewritten as

$$
\frac{3 h^{2}\left(4-C_{\alpha} \tau^{\alpha}\right)}{h^{2}+4^{1-\alpha} \tau^{\alpha}\left(12 K_{\alpha}-C_{\alpha} h^{2}\right)} \geq 4,
$$

where $K_{\alpha}>0$ and $C_{\alpha}>0$ are diffusion and reaction coefficients, respectively.

In addition, the numerical check of the validity of the stability condition (83) will be discussed in Section 4.

Moreover, from Theorems 8 and 11, we know that Numerical Algorithm I (see (38)) and Numerical Algorithm II (see (61)) are all consistent with the local truncation errors 


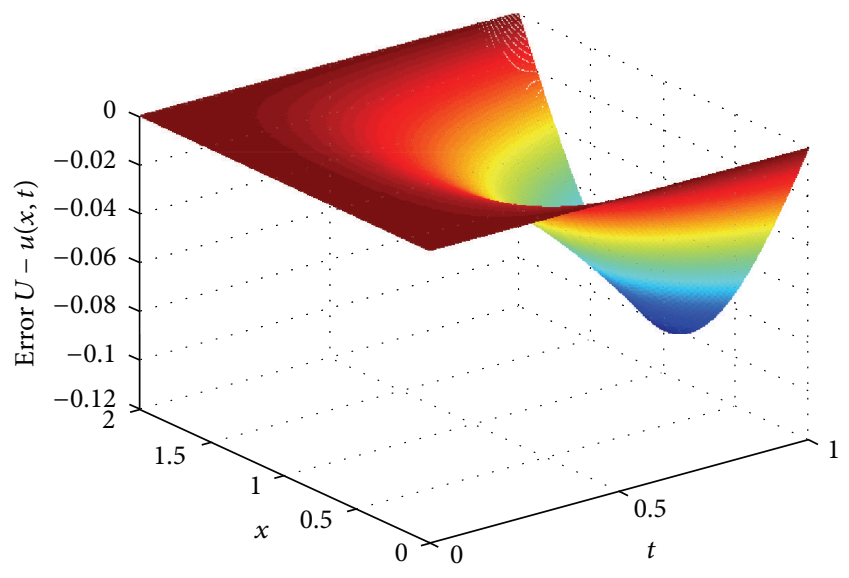

FIGURE 3: The error of the analytical solution and the numerical solution for $\alpha=1.5$ by Numerical Algorithm I (see (38)) with $\tau=0.0025, h=0.01$.

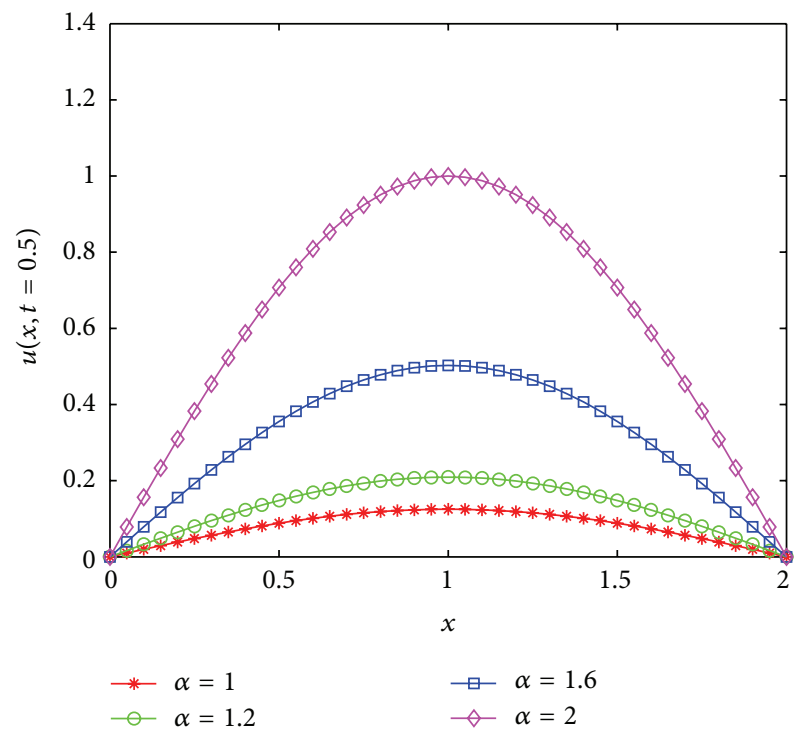

FIGURE 4: Dependence of the numerical solution for different values of $\alpha$ by Numerical Algorithm II (see (61)) with $\tau=0.002, h=0.0025$.

$\widehat{R}_{l}^{k}=\mathcal{O}\left(\tau^{2}+h^{4}\right)$ and $\widetilde{R}_{l}^{k}=\mathcal{O}\left(\tau^{2}+h^{4}\right)$ because $\widehat{R}_{l}^{k}, \widetilde{R}_{l}^{k} \rightarrow 0$ as $\tau, h \rightarrow 0$. Therefore, according to Lax's Equivalence Theorem [33], Numerical Algorithm I unconditionally converges at the same order $\mathcal{O}\left(\tau^{2}+h^{4}\right)$, but Numerical Algorithm II converges at the same order $\mathcal{O}\left(\tau^{2}+h^{4}\right)$ under condition (83).

\section{Numerical Example}

In this section, we give a numerical example to demonstrate the efficiency of the derived numerical algorithms.

Example 1. Consider the following equation:

$$
\begin{array}{r}
{ }_{C} D_{0, t}^{\alpha} u(x, t)=\frac{\partial^{2} u(x, t)}{\partial x^{2}}-u(x, t)+f(x, t), \\
0 \leq t \leq 1,0<x<2,
\end{array}
$$

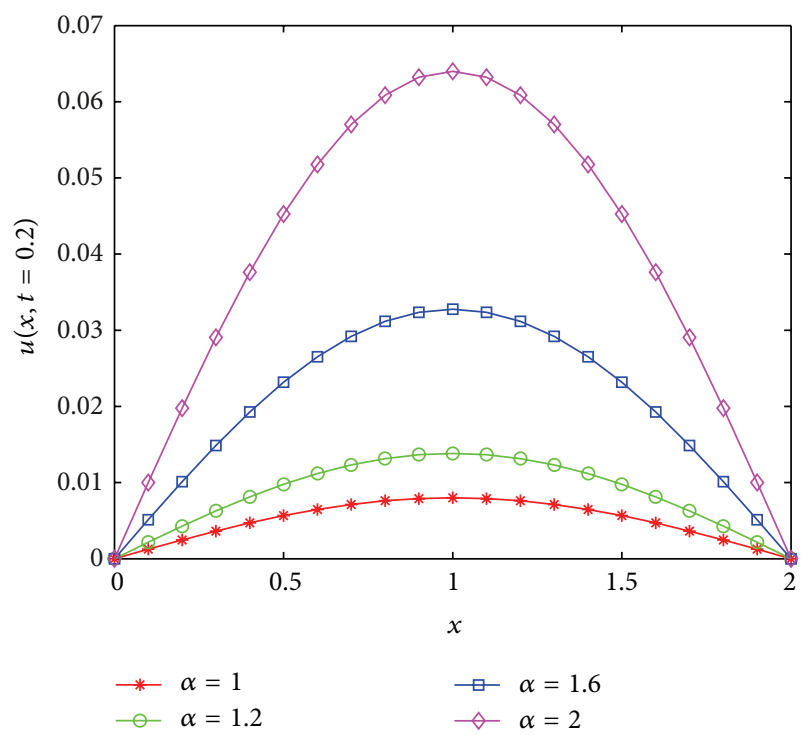

FIGURE 5: Dependence of the numerical solution for different values of $\alpha$ by Numerical Algorithm II (see (61)) with $\tau=0.0001, h=0.05$.

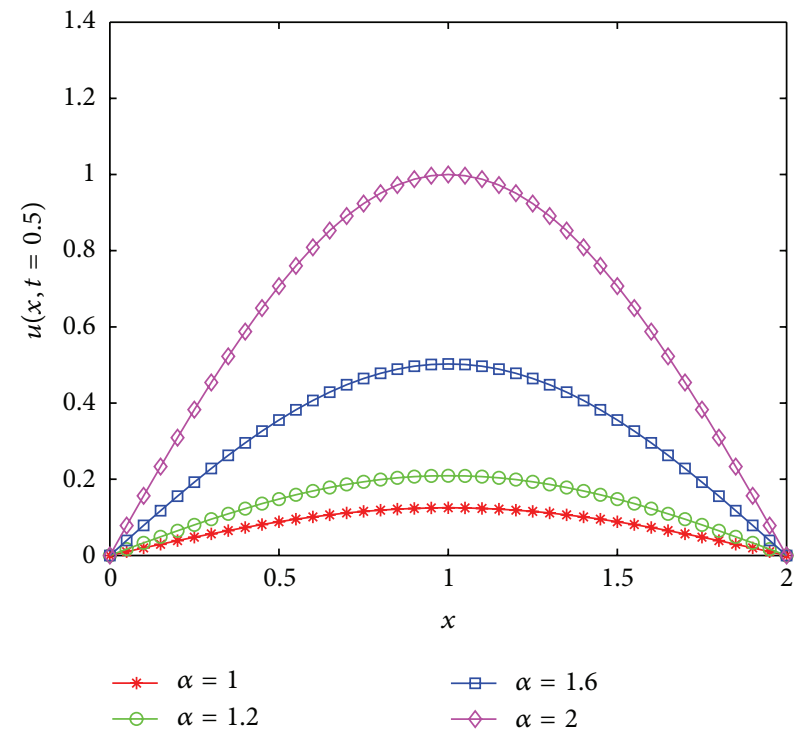

FIGURE 6: The error of the analytical solution and the numerical solution for $\alpha=1.5$ by Numerical Algorithm II (see (61)) with $\tau=0.04, h=0.002$.

where $f(x, t)=\alpha^{3}\left[\left(6 t^{3-\alpha} / \Gamma(4-\alpha)\right)+t^{3}\left(1+\left(\pi^{2} / 4\right)\right)\right] \sin ((\pi /$ 2)x).

The analytical solution of this equation is $u(x, t)=$ $(\alpha t)^{3} \sin ((\pi / 2) x)$. The initial conditions and the boundary conditions are satisfied with the exact solution $u(x, t)$ given previously.

On one hand, we use Numerical Algorithm I (see (38)) to solve the previous equation. At first, we give the comparisons of the analytical and numerical solutions for different order, $\alpha, \tau$, and $h$ in Figures 1 and 2, respectively, and we observe that the numerical solution is in line with the analytical solution 


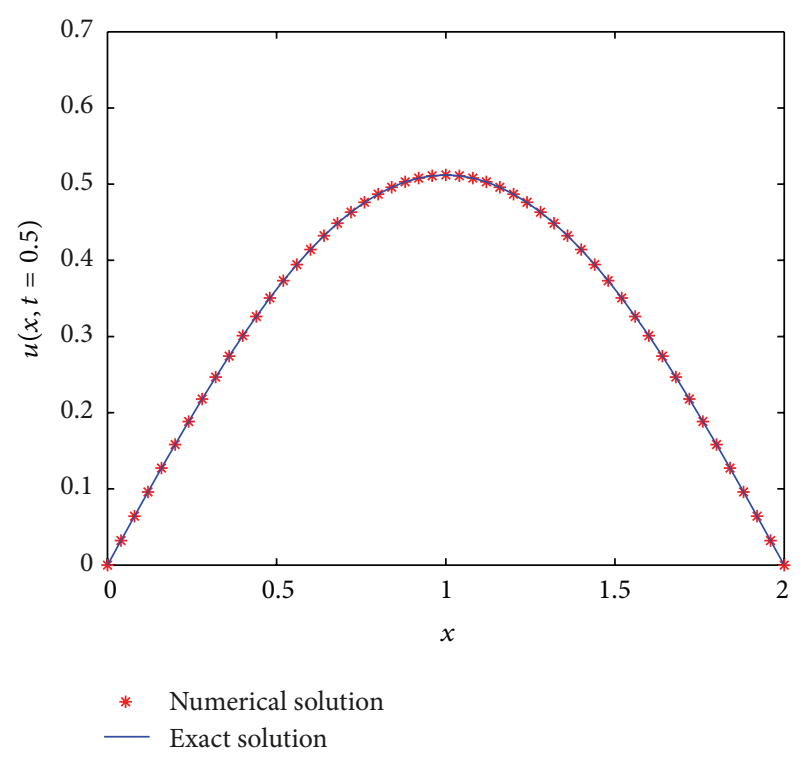

Figure 7: Comparison of the analytical solution and numerical solution for $\alpha=1.6$ by Numerical Algorithm II (see (61)) with $h=0.04, \tau=0.005$, which satisfy the stability (83).

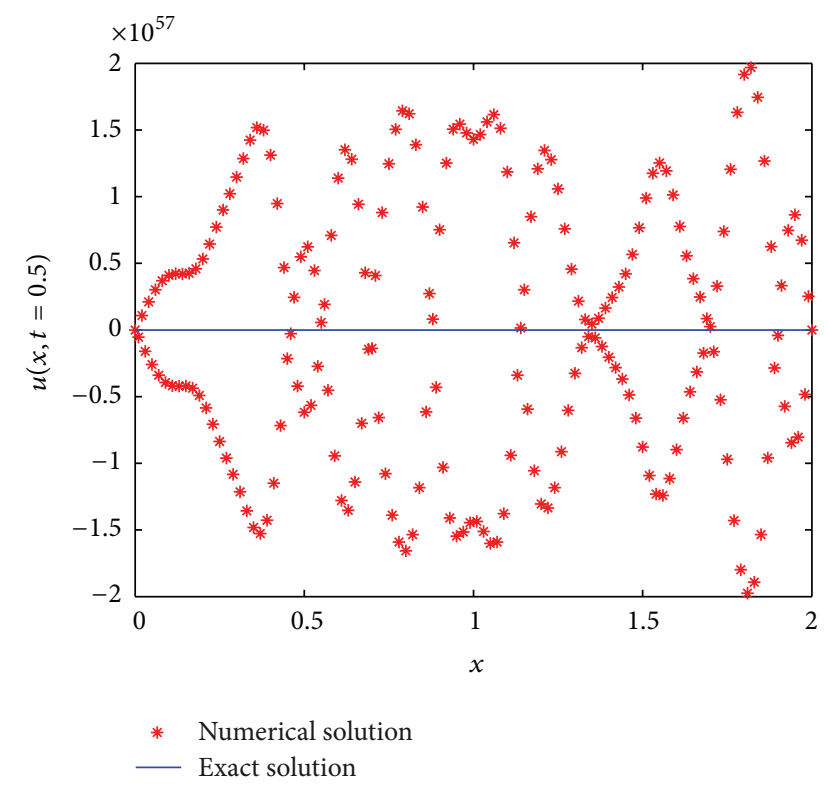

FIGURE 8: Comparison of the analytical solution and numerical solution for $\alpha=1.6$ by Numerical Algorithm II (see (61)) with $h=0.01, \tau=0.01$, which do not satisfy the stability (83).

and the velocity $u$ increases with the increase of order $\alpha$. Meanwhile, the errors are shown in Figure 3 with $\alpha=1.5$. Finally, the temporal and spatial convergence orders are listed in Table 1.

Next, we use Numerical Algorithm II (61). In this situation, we take (60), the equivalence equation of (1), where $g(x, t)=6 t \alpha^{3}\left[1+\left(t^{\alpha} / \Gamma(2+\alpha)\right)\left(1+\left(\pi^{2} / 4\right)\right)\right] \sin ((\pi / 2) x)$.

Figures 4 and 5 display that the velocity $u$ increases with the increase of order $\alpha$ for different $\tau$ and $h$. Figure 6 presents the error between the analytical solution and numerical solution of (60). Table 2 lists the temporal convergence orders.

Finally, we checked the stability condition given in (83) in the following way. Firstly, we choose $h=0.04, \tau=$ 0.005 , and $\alpha=1.6$, which satisfy stability condition (83), the comparison result of the numerical solution and analytical solution is shown in Figure 7. Secondly, we choose $h=$ $0.01, \tau=0.01$, and $\alpha=1.6$, and then these parameters do not satisfy stability condition (83); the comparison result of the numerical solution and analytical solution is shown in Figure 8. From Figures 7 and 8, we declare that stability condition (83) is valid.

\section{Conclusion}

In this paper, we construct two difference schemes for solving the fractional diffusion-wave equation with reaction term. It is proved that Numerical Algorithm I (38) is unconditionally stable and Numerical Algorithm II (61) is conditionally stable by using the fractional Fourier method. The local truncation errors of two difference schemes are both $\mathcal{O}\left(\tau^{2}+h^{4}\right)$, which are the best results till now. Finally, the numerical results given in this paper show the effectiveness of the derived numerical algorithms.

\section{Acknowledgments}

The work was partially supported by the Key Program of Shanghai Municipal Education Commission under Grant no. 12ZZ084 and the grant of "The First-Class Discipline of Universities in Shanghai."

\section{References}

[1] E. Barkai, R. Metzler, and J. Klafter, "From continuous time random walks to the fractional Fokker-Planck equation," Physical Review E, vol. 61, no. 1, pp. 132-138, 2000.

[2] D. A. Benson, S. W. Wheatcraft, and M. M. Meerschaert, "Application of a fractional advection-dispersion equation," Water Resources Research, vol. 36, no. 6, pp. 1403-1412, 2000.

[3] R. Hilfer, Applications of Fractional Calculus in Physics, World Scientific, Singapore, 2000.

[4] F. Mainardi, "Fractional calculus: some basic problems in continuum and statistical mechanics," in Fractals and Fractional Calculus in Continuum Mechanics, A. Carpinteri and F. Mainardi, Eds., Springer, New York, NY, USA, 1997.

[5] K. S. Miller and B. Ross, An Introduction to the Fractional Calculus and Fractional Differential Equations, John Wiley \& Sons, New York, NY, USA, 1993.

[6] A. I. Saichev and G. M. Zaslavsky, "Fractional kinetic equations: solutions and applications," Chaos, vol. 7, no. 4, pp. 753-764, 1997.

[7] S. B. Yuste, L. Acedo, and K. Lindenberg, "Reaction front in an $\mathrm{A}+\mathrm{B} \rightarrow \mathrm{C}$ reactionsubdiffusion process," Physical Review E, vol. 69, no. 3, part 2, Article ID 036126, 2004.

[8] S. B. Yuste and K. Lindenberg, "Subdiffusion-limited A + A reactions," Physical Review Letters, vol. 87, no. 11, Article ID 118301, 2001. 
[9] M. Cui, "Compact finite difference method for the fractional diffusion equation," Journal of Computational Physics, vol. 228, no. 20, pp. 7792-7804, 2009.

[10] C.-M. Chen, F. Liu, I. Turner, and V. Anh, "A Fourier method for the fractional diffusion equation describing sub-diffusion," Journal of Computational Physics, vol. 227, no. 2, pp. 886-897, 2007.

[11] C. P. Li, A. Chen, and J. J. Ye, "Numerical approaches to fractional calculus and fractional ordinary differential equation," Journal of Computational Physics, vol. 230, no. 9, pp. 3352-3368, 2011.

[12] F. Liu, C. Yang, and K. Burrage, "Numerical method and analytic technique of the modified anomalous subdiffusion equation with a nonlinear source term," Journal of Computational and Applied Mathematics, vol. 231, no. 1, pp. 160-176, 2009.

[13] E. Sousa, "Finite difference approximations for a fractional advection diffusion problem," Journal of Computational Physics, vol. 228, no. 11, pp. 4038-4054, 2009.

[14] E. Sousa, "Numerical approximations for fractional diffusion equations via splines," Computers \& Mathematics with Applications, vol. 62, no. 3, pp. 938-944, 2011.

[15] H. Wang and K. Wang, "An $O\left(N \log ^{2} N\right)$ alternating-direction finite difference method for two-dimensional fractional diffusion equations," Journal of Computational Physics, vol. 230, no. 21, pp. 7830-7839, 2011.

[16] S. B. Yuste and L. Acedo, "An explicit finite difference method and a new von Neumann-type stability analysis for fractional diffusion equations," SIAM Journal on Numerical Analysis, vol. 42, no. 5, pp. 1862-1874, 2005.

[17] V. J. Ervin, N. Heuer, and J. P. Roop, "Numerical approximation of a time dependent, nonlinear, space-fractional diffusion equation," SIAM Journal on Numerical Analysis, vol. 45, no. 2, pp. 572-591, 2007.

[18] G. J. Fix and J. P. Roop, "Least squares finite-element solution of a fractional order two-point boundary value problem," Computers \& Mathematics with Applications, vol. 48, no. 7-8, pp. 1017-1033, 2004.

[19] J. P. Roop, "Computational aspects of FEM approximation of fractional advection dispersion equations on bounded domains in $R^{2}$," Journal of Computational and Applied Mathematics, vol. 193, no. 1, pp. 243-268, 2006.

[20] C. P. Li, Z. G. Zhao, and Y. Q. Chen, "Numerical approximation of nonlinear fractional differential equations with subdiffusion and superdiffusion," Computers \& Mathematics with Applications, vol. 62, no. 3, pp. 855-875, 2011.

[21] H. Zhang, F. Liu, and V. Anh, "Galerkin finite element approximation of symmetric space-fractional partial differential equations," Applied Mathematics and Computation, vol. 217, no. 6, pp. 2534-2545, 2010.

[22] Y. Y. Zheng, C. P. Li, and Z. G. Zhao, "A fully discrete discontinuous Galerkin method for nonlinear fractional FokkerPlanck equation," Mathematical Problems in Engineering, vol. 2010, Article ID 279038, 26 pages, 2010.

[23] Q. Yang, F. Liu, and I. Turner, "Numerical methods for fractional partial differential equations with Riesz space fractional derivatives," Applied Mathematical Modelling, vol. 34, no. 1, pp. 200-218, 2010.

[24] Q. Yang, F. Liu, and I. Turner, "Computationally efficient numerical methods for time and space fractional Fokker-Planck equations," Physica Scripta, vol. 2009, Article ID 014026, 2009.
[25] C. P. Li, F. H. Zeng, and F. Liu, "Spectral approximations to the fractional integral and derivative," Fractional Calculus and Applied Analysis, vol. 15, no. 3, pp. 383-406, 2012.

[26] X. Li and C. Xu, "A space-time spectral method for the time fractional diffusion equation," SIAM Journal on Numerical Analysis, vol. 47, no. 3, pp. 2108-2131, 2009.

[27] H. F. Ding and C. P. Li, "Mixed spline function method for reaction-subdiusion equations," Journal of Computational Physics, vol. 242, pp. 103-123, 2013.

[28] F. Mainardi, "Fractional relaxation-oscillation and fractional diffusion-wave phenomena," Chaos, Solitons and Fractals, vol. 7, no. 9, pp. 1461-1477, 1996.

[29] J. Q. Murillo and S. B. Yuste, "An explicit difference method for solving fractional diffusion and diffusion-wave equations in the Caputo form," Journal of Computational and Nonlinear Dynamics, vol. 6, no. 2, 6 pages, 2011.

[30] I. Podlubny, Fractional Differential Equations, Academic Press, San Diego, Calif, USA, 1999.

[31] D. K. Salkuyeh, "On the finite difference approximation to the convection-diffusion equation," Applied Mathematics and Computation, vol. 179, no. 1, pp. 79-86, 2006.

[32] M. Li, T. Tang, and B. Fornberg, "A compact fourth-order finite difference scheme for the steady incompressible NavierStokes equations," International Journal for Numerical Methods in Fluids, vol. 20, no. 10, pp. 1137-1151, 1995.

[33] L. Su, W. Wang, and Q. Xu, "Finite difference methods for fractional dispersion equations," Applied Mathematics and Computation, vol. 216, no. 11, pp. 3329-3334, 2010. 


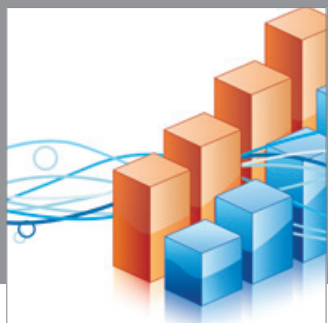

Advances in

Operations Research

mansans

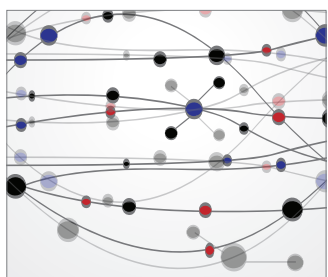

The Scientific World Journal
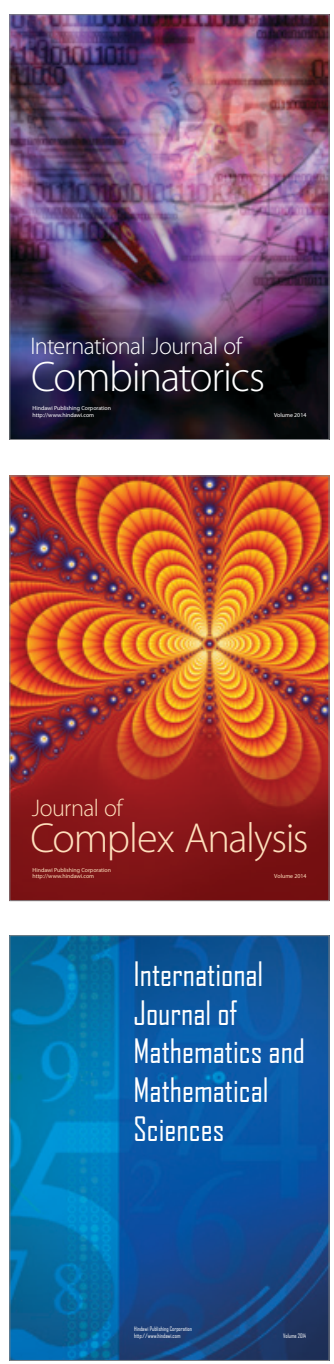
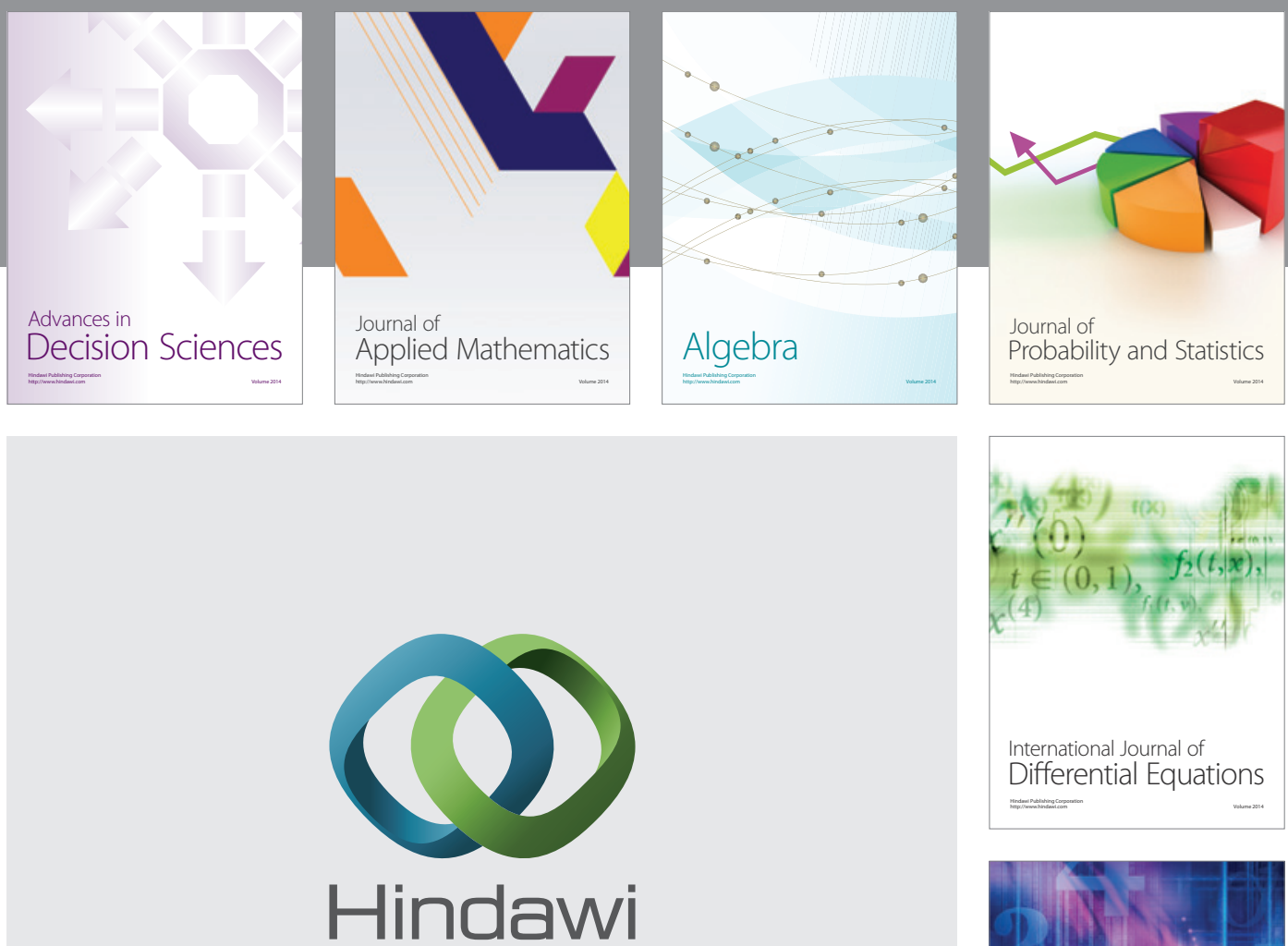

Submit your manuscripts at http://www.hindawi.com
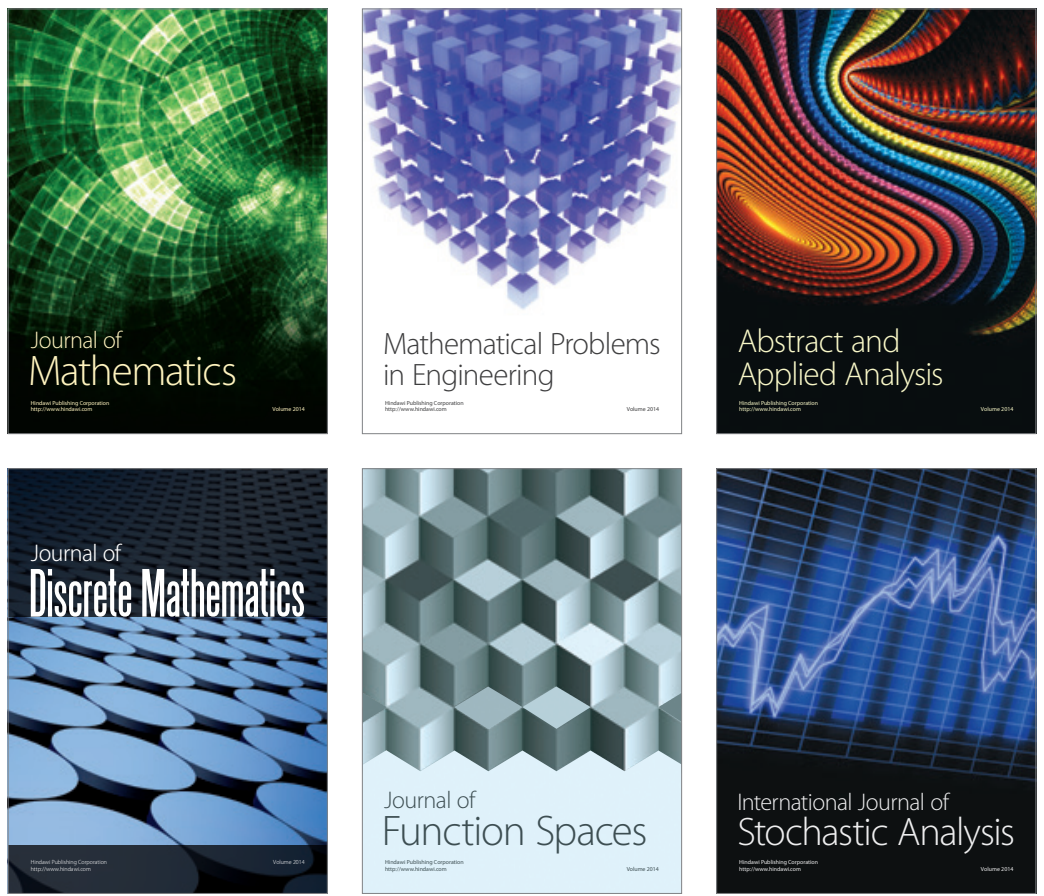

Journal of

Function Spaces

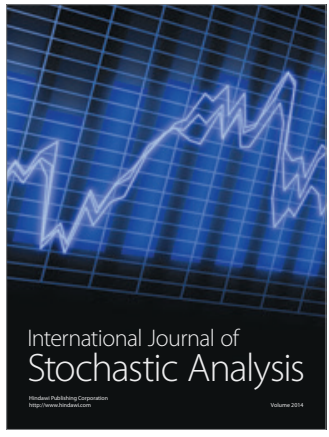

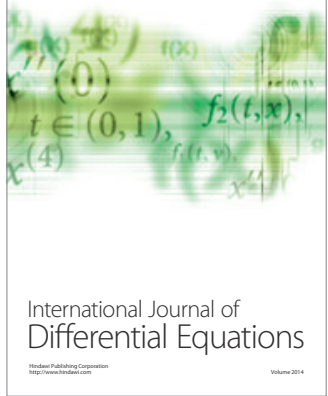
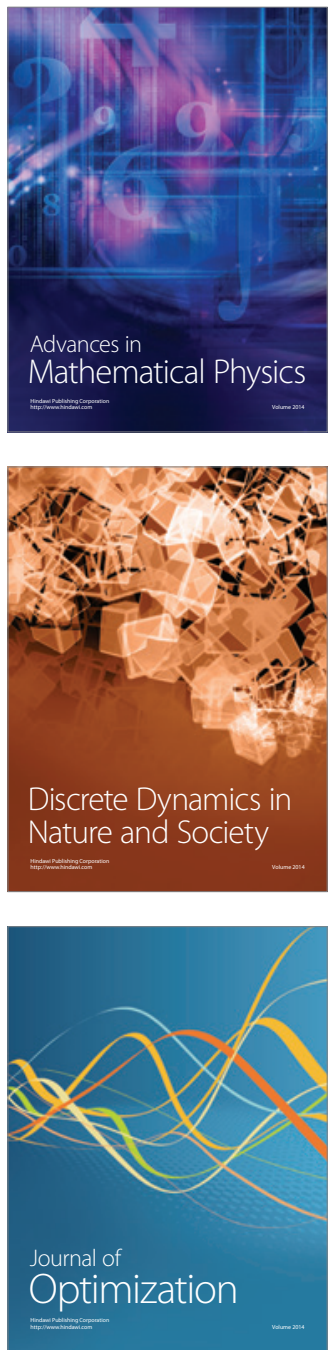